\title{
Quantitative symplectic geometry
}

\author{
KAI CIELIEBAK, HELMUT HOFER, \\ JANKO LATSCHEV, AND FELIX SCHLENK
}

\section{Dedicated to Anatole Katok on the occasion of his sixtieth birthday}

A symplectic manifold $(M, \omega)$ is a smooth manifold $M$ endowed with a nondegenerate and closed 2-form $\omega$. By Darboux's Theorem such a manifold looks locally like an open set in some $\mathbb{R}^{2 n} \cong \mathbb{C}^{n}$ with the standard symplectic form

$$
\omega_{0}=\sum_{j=1}^{n} d x_{j} \wedge d y_{j},
$$

and so symplectic manifolds have no local invariants. This is in sharp contrast to Riemannian manifolds, for which the Riemannian metric admits various curvature invariants. Symplectic manifolds do however admit many global numerical invariants, and prominent among them are the so-called symplectic capacities.

Symplectic capacities were introduced in 1990 by I. Ekeland and H. Hofer [18; 19] (although the first capacity was in fact constructed by M. Gromov [39]). Since then, lots of new capacities have been defined $[16 ; 29 ; 31 ; 43 ; 48 ; 58$; $59 ; 88 ; 97]$ and they were further studied in $[1 ; 2 ; 8 ; 9 ; 25 ; 20 ; 27 ; 30 ; 34$; $36 ; 37 ; 40 ; 41 ; 42 ; 45 ; 47 ; 49 ; 51 ; 55 ; 56 ; 57 ; 60 ; 61 ; 62 ; 63 ; 65 ; 71 ; 72$; $73 ; 86 ; 87 ; 89 ; 90 ; 92 ; 95 ; 96]$. Surveys on symplectic capacities are $[44 ; 49$; 54; 66; 95]. Different capacities are defined in different ways, and so relations between capacities often lead to surprising relations between different aspects of symplectic geometry and Hamiltonian dynamics. This is illustrated in Section 2, where we discuss some examples of symplectic capacities and describe a few consequences of their existence. In Section 3 we present an attempt to

Cieliebak's research was partially supported by the DFG grant Ci 45/2-1. Hofer's research was partially supported by the NSF Grant DMS-0102298. Latschev held a position financed by the DFG grant Mo 843/2-1. Schlenk held a position financed by the DFG grant Schw 892/2-1. 
better understand the space of all symplectic capacities, and discuss some further general properties of symplectic capacities. In Section 4, we describe several new relations between certain symplectic capacities on ellipsoids and polydiscs. Throughout the discussion we mention many open problems.

As illustrated below, many of the quantitative aspects of symplectic geometry can be formulated in terms of symplectic capacities. Of course there are other numerical invariants of symplectic manifolds which could be included in a discussion of quantitative symplectic geometry, such as the invariants derived from Hofer's bi-invariant metric on the group of Hamiltonian diffeomorphisms, [43; $79 ; 82$ ], or Gromov-Witten invariants. Their relation to symplectic capacities is not well understood, and we will not discuss them here.

We start out with a brief description of some relations of symplectic geometry to neighboring fields.

\section{Symplectic geometry and its neighbors}

Symplectic geometry is a rather new and vigorously developing mathematical discipline. The "symplectic explosion" is described in [21]. Examples of symplectic manifolds are open subsets of $\left(\mathbb{R}^{2 n}, \omega_{0}\right)$, the torus $\mathbb{R}^{2 n} / \mathbb{Z}^{2 n}$ endowed with the induced symplectic form, surfaces equipped with an area form, Kähler manifolds like complex projective space $\mathbb{C P}^{n}$ endowed with their Kähler form, and cotangent bundles with their canonical symplectic form. Many more examples are obtained by taking products and through more elaborate constructions, such as the symplectic blow-up operation. A diffeomorphism $\varphi$ on a symplectic manifold $(M, \omega)$ is called symplectic or a symplectomorphism if $\varphi^{*} \omega=\omega$.

A fascinating feature of symplectic geometry is that it lies at the crossroad of many other mathematical disciplines. In this section we mention a few examples of such interactions.

Hamiltonian dynamics. Symplectic geometry originated in Hamiltonian dynamics, which originated in celestial mechanics. A time-dependent Hamiltonian function on a symplectic manifold $(M, \omega)$ is a smooth function $H: \mathbb{R} \times M \rightarrow \mathbb{R}$. Since $\omega$ is nondegenerate, the equation

$$
\omega\left(X_{H}, \cdot\right)=d H(\cdot)
$$

defines a time-dependent smooth vector field $X_{H}$ on $M$. Under suitable assumption on $H$, this vector field generates a family of diffeomorphisms $\varphi_{H}^{t}$ called the Hamiltonian flow of $H$. As is easy to see, each map $\varphi_{H}^{t}$ is symplectic. A Hamiltonian diffeomorphism $\varphi$ on $M$ is a diffeomorphism of the form $\varphi_{H}^{1}$. 
Symplectic geometry is the geometry underlying Hamiltonian systems. It turns out that this geometric approach to Hamiltonian systems is very fruitful. Explicit examples are discussed in Section 2 below.

Volume geometry. A volume form $\Omega$ on a manifold $M$ is a top-dimensional nowhere vanishing differential form, and a diffeomorphism $\varphi$ of $M$ is volume preserving if $\varphi^{*} \Omega=\Omega$. Ergodic theory studies the properties of volume preserving mappings. Its findings apply to symplectic mappings. Indeed, since a symplectic form $\omega$ is nondegenerate, $\omega^{n}$ is a volume form, which is preserved under symplectomorphisms. In dimension 2 a symplectic form is just a volume form, so that a symplectic mapping is just a volume preserving mapping. In dimensions $2 n \geq 4$, however, symplectic mappings are much more special. A geometric example for this is Gromov's Nonsqueezing Theorem stated in Section 2.2 and a dynamical example is the (partly solved) Arnol'd conjecture stating that Hamiltonian diffeomorphisms of closed symplectic manifolds have at least as many fixed points as smooth functions have critical points. For another link between ergodic theory and symplectic geometry see [81].

Contact geometry. Contact geometry originated in geometrical optics. A contact manifold $(P, \alpha)$ is a $(2 n-1)$-dimensional manifold $P$ endowed with a 1 -form $\alpha$ such that $\alpha \wedge(d \alpha)^{n-1}$ is a volume form on $P$. The vector field $X$ on $P$ defined by $d \alpha(X, \cdot)=0$ and $\alpha(X)=1$ generates the so-called Reeb flow. The restriction of a time-independent Hamiltonian system to an energy surface can sometimes be realized as the Reeb flow on a contact manifold. Contact manifolds also arise naturally as boundaries of symplectic manifolds. One can study a contact manifold $(P, \alpha)$ by symplectic means by looking at its symplectization $\left(P \times \mathbb{R}, d\left(e^{t} \alpha\right)\right)$, see e.g. [46; 22].

Algebraic geometry. A special class of symplectic manifolds are Kähler manifolds. Such manifolds (and, more generally, complex manifolds) can be studied by looking at holomorphic curves in them. M. Gromov [39] observed that some of the tools used in the Kähler context can be adapted for the study of symplectic manifolds. One part of his pioneering work has grown into what is now called Gromov-Witten theory, see e.g. [70] for an introduction.

Many other techniques and constructions from complex geometry are useful in symplectic geometry. For example, there is a symplectic version of blowingup, which is intimately related to the symplectic packing problem, see $[64 ; 68]$ and 4.1.2 below. Another example is Donaldson's construction of symplectic submanifolds [17]. Conversely, symplectic techniques proved useful for studying problems in algebraic geometry such as Nagata's conjecture $[5 ; 6 ; 68]$ and degenerations of algebraic varieties [7]. 
Riemannian and spectral geometry. Recall that the differentiable structure of a smooth manifold $M$ gives rise to a canonical symplectic form on its cotangent bundle $T^{*} M$. Giving a Riemannian metric $g$ on $M$ is equivalent to prescribing its unit cosphere bundle $S_{g}^{*} M \subset T^{*} M$, and the restriction of the canonical 1-form from $T^{*} M$ gives $S^{*} M$ the structure of a contact manifold. The Reeb flow on $S_{g}^{*} M$ is the geodesic flow (free particle motion).

In a somewhat different direction, each symplectic form $\omega$ on some manifold $M$ distinguishes the class of Riemannian metrics which are of the form $\omega(J \cdot, \cdot)$ for some almost complex structure $J$.

These (and other) connections between symplectic and Riemannian geometry are by no means completely explored, and we believe there is still plenty to be discovered here. Here are some examples of known results relating Riemannian and symplectic aspects of geometry.

Lagrangian submanifolds. A middle-dimensional submanifold $L$ of $(M, \omega)$ is called Lagrangian if $\omega$ vanishes on $T L$.

(i) Volume. Endow complex projective space $\mathbb{C P}^{n}$ with the usual Kähler metric and the usual Kähler form. The volume of submanifolds is taken with respect to this Riemannian metric. According to a result of Givental-KleinerOh, the standard $\mathbb{R} \mathbb{P}^{n}$ in $\mathbb{C P}^{n}$ has minimal volume among all its Hamiltonian deformations [74]. A partial result for the Clifford torus in $\mathbb{C P}^{n}$ can be found in [38]. The torus $S^{1} \times S^{1} \subset S^{2} \times S^{2}$ formed by the equators is also volume minimizing among its Hamiltonian deformations, [50]. If $L$ is a closed Lagrangian submanifold of $\left(\mathbb{R}^{2 n}, \omega_{0}\right)$, there exists according to [98] a constant $C$ depending on $L$ such that

$$
\operatorname{vol}\left(\varphi_{H}(L)\right) \geq C \quad \text { for all Hamiltonian deformations of } L
$$

(ii) Mean curvature. The mean curvature form of a Lagrangian submanifold $L$ in a Kähler-Einstein manifold can be expressed through symplectic invariants of $L$, see [15].

The first eigenvalue of the Laplacian. Symplectic methods can be used to estimate the first eigenvalue of the Laplace operator on functions for certain Riemannian manifolds [80].

Short billiard trajectories. Consider a bounded domain $U \subset \mathbb{R}^{n}$ with smooth boundary. There exists a periodic billiard trajectory on $\bar{U}$ of length $l$ with

$$
l^{n} \leq C_{n} \operatorname{vol}(U)
$$

where $C_{n}$ is an explicit constant depending only on $n$, see $[98 ; 30]$. 


\section{Examples of symplectic capacities}

In this section we give the formal definition of symplectic capacities, and discuss a number of examples along with sample applications.

2.1. Definition. Denote by $S y m p^{2 n}$ the category of all symplectic manifolds of dimension $2 n$, with symplectic embeddings as morphisms. A symplectic category is a subcategory $\mathscr{C}$ of $\operatorname{Symp}^{2 n}$ such that $(M, \omega) \in \mathscr{C}$ implies $(M, \alpha \omega) \in \mathscr{C}$ for all $\alpha>0$.

CONVENTION. We will use the symbol $\hookrightarrow$ to denote symplectic embeddings and $\rightarrow$ to denote morphisms in the category $\mathscr{b}$ (which may be more restrictive).

Let $B^{2 n}\left(r^{2}\right)$ be the open ball of radius $r$ in $\mathbb{R}^{2 n}$ and $Z^{2 n}\left(r^{2}\right)=B^{2}\left(r^{2}\right) \times \mathbb{R}^{2 n-2}$ the open cylinder (the reason for this notation will become apparent below). Unless stated otherwise, open subsets of $\mathbb{R}^{2 n}$ are always equipped with the canonical symplectic form $\omega_{0}=\sum_{j=1}^{n} d y_{j} \wedge d x_{j}$. We will suppress the dimension $2 n$ when it is clear from the context and abbreviate

$$
B:=B^{2 n}(1), \quad Z:=Z^{2 n}(1) .
$$

Now let $\mathscr{C} \subset S_{y m p}{ }^{2 n}$ be a symplectic category containing the ball $B$ and the cylinder $Z$. A symplectic capacity on $\mathscr{C}$ is a covariant functor $c$ from $\mathscr{C}$ to the category $([0, \infty], \leq)$ (with $a \leq b$ as morphisms) satisfying

(Monotonicity): $c(M, \omega) \leq c\left(M^{\prime}, \omega^{\prime}\right)$ if there exists a morphism $(M, \omega) \rightarrow$ $\left(M^{\prime}, \omega^{\prime}\right)$;

(CONFORMALiTy): $c(M, \alpha \omega)=\alpha c(M, \omega)$ for $\alpha>0$;

(Nontriviality): $0<c(B)$ and $c(Z)<\infty$.

Note that the (Monotonicity) axiom just states the functoriality of $c$. A symplectic capacity is said to be normalized if

$($ NORMALIZATION): $c(B)=1$.

As a frequent example we will use the set $O p^{2 n}$ of open subsets in $\mathbb{R}^{2 n}$. We make it into a symplectic category by identifying $\left(U, \alpha^{2} \omega_{0}\right)$ with the symplectomorphic manifold $\left(\alpha U, \omega_{0}\right)$ for $U \subset \mathbb{R}^{2 n}$ and $\alpha>0$. We agree that the morphisms in this category shall be symplectic embeddings induced by global symplectomorphisms of $\mathbb{R}^{2 n}$. With this identification, the (Conformality) axiom above takes the form

(CONFORMALity)': $c(\alpha U)=\alpha^{2} c(U)$ for $U \in O p^{2 n}, \alpha>0$. 
2.2. Gromov radius. In view of Darboux's Theorem one can associate with each symplectic manifold $(M, \omega)$ the numerical invariant

$$
c_{B}(M, \omega):=\sup \left\{\alpha>0 \mid B^{2 n}(\alpha) \hookrightarrow(M, \omega)\right\}
$$

called the Gromov radius of $(M, \omega)$, [39]. It measures the symplectic size of $(M, \omega)$ in a geometric way, and is reminiscent of the injectivity radius of a Riemannian manifold. Note that it clearly satisfies the (Monotonicity) and (Conformality) axioms for a symplectic capacity. It is equally obvious that $c_{B}(B)=1$.

If $M$ is 2-dimensional and connected, then $\pi c_{B}(M, \omega)=\int_{M} \omega$, i.e. $c_{B}$ is proportional to the volume of $M$, see [89]. The following theorem from Gromov's seminal paper [39] implies that in higher dimensions the Gromov radius is an invariant very different from the volume.

NONSQUEEZING THEOREM (GROMOV, 1985). The cylinder $Z \in$ Symp $^{2 n}$ satisfies $c_{B}(Z)=1$.

Therefore the Gromov radius is a normalized symplectic capacity on $S y m p^{2 n}$. Gromov originally obtained this result by studying properties of moduli spaces of pseudo-holomorphic curves in symplectic manifolds.

It is important to realize that the existence of at least one capacity $c$ with $c(B)=c(Z)$ also implies the Nonsqueezing Theorem. We will see below that each of the other important techniques in symplectic geometry (such as variational methods and the global theory of generating functions) gave rise to the construction of such a capacity, and hence an independent proof of this fundamental result.

It was noted in [18] that the following result, originally established by Eliashberg and by Gromov using different methods, is also an easy consequence of the existence of a symplectic capacity.

THEOREM (ELIASHBERG, GROMOV). The group of symplectomorphisms of a symplectic manifold $(M, \omega)$ is closed for the compact-open $C^{0}$-topology in the group of all diffeomorphisms of $M$.

2.3. Symplectic capacities via Hamiltonian systems. The next four examples of symplectic capacities are constructed via Hamiltonian systems. A crucial role in the definition or the construction of these capacities is played by the action functional of classical mechanics. For simplicity, we assume that $(M, \omega)=$ $\left(\mathbb{R}^{2 n}, \omega_{0}\right)$. Given a Hamiltonian function $H: S^{1} \times \mathbb{R}^{2 n} \rightarrow \mathbb{R}$ which is periodic in the time-variable $t \in S^{1}=\mathbb{R} / \mathbb{Z}$ and which generates a global flow $\varphi_{H}^{t}$, the 
action functional on the loop space $C^{\infty}\left(S^{1}, \mathbb{R}^{2 n}\right)$ is defined as

$$
\mathscr{A}_{H}(\gamma)=\int_{\gamma} y d x-\int_{0}^{1} H(t, \gamma(t)) d t .
$$

Its critical points are exactly the 1-periodic orbits of $\varphi_{H}^{t}$. Since the action functional is neither bounded from above nor from below, critical points are saddle points. In his pioneering work [83; 84], P. Rabinowitz designed special minimax principles adapted to the hyperbolic structure of the action functional to find such critical points. We give a heuristic argument why this works. Consider the space of loops

$$
E=H^{1 / 2}\left(S^{1}, \mathbb{R}^{2 n}\right)=\left\{\left.z \in L^{2}\left(S^{1} ; \mathbb{R}^{2 n}\right)\left|\sum_{k \in \mathbb{Z}}\right| k|| z_{k}\right|^{2}<\infty\right\}
$$

where $z=\sum_{k \in \mathbb{Z}} e^{2 \pi k t J} z_{k}, z_{k} \in \mathbb{R}^{2 n}$, is the Fourier series of $z$ and $J$ is the standard complex structure of $\mathbb{R}^{2 n} \cong \mathbb{C}^{n}$. The space $E$ is a Hilbert space with inner product

$$
\langle z, w\rangle=\left\langle z_{0}, w_{0}\right\rangle+2 \pi \sum_{k \in \mathbb{Z}}|k|\left\langle z_{k}, w_{k}\right\rangle,
$$

and there is an orthogonal splitting $E=E^{-} \oplus E^{0} \oplus E^{+}, z=z^{-}+z^{0}+z^{+}$, into the spaces of $z \in E$ having nonzero Fourier coefficients $z_{k} \in \mathbb{R}^{2 n}$ only for $k<0, k=0, k>0$. The action functional $\mathscr{A}_{H}: C^{\infty}\left(S^{1}, \mathbb{R}^{2 n}\right) \rightarrow \mathbb{R}$ extends to $E$ as

$$
\mathscr{A}_{H}(z)=\left(\frac{1}{2}\left\|z^{+}\right\|^{2}-\frac{1}{2}\left\|z^{-}\right\|^{2}\right)-\int_{0}^{1} H(t, z(t)) d t .
$$

Notice now the hyperbolic structure of the first term $\mathscr{A}_{0}(x)$, and that the second term is of lower order. Some of the critical points $z(t) \equiv$ const of $\mathscr{A}_{0}$ should thus persist for $H \neq 0$.

2.3.1. Ekeland-Hofer capacities. The first construction of symplectic capacities via Hamiltonian systems was carried out by Ekeland and Hofer [18; 19]. To give the heuristics, we consider a bounded domain $U \subset \mathbb{R}^{2 n}$ with smooth boundary $\partial U$. A closed characteristic $\gamma$ on $\partial U$ is an embedded circle in $\partial U$ tangent to the characteristic line bundle

$$
\mathscr{L}_{U}=\left\{(x, \xi) \in T \partial U \mid \omega_{0}(\xi, \eta)=0 \text { for all } \eta \in T_{x} \partial U\right\} .
$$

If $\partial U$ is represented as a regular energy surface $\left\{x \in \mathbb{R}^{2 n} \mid H(x)=\right.$ const $\}$ of a smooth function $H$ on $\mathbb{R}^{2 n}$, then the Hamiltonian vector field $X_{H}$ restricted to $\partial U$ is a section of $\mathscr{L}_{U}$, and so the traces of the periodic orbits of $X_{H}$ on $\partial U$ are 
the closed characteristics on $\partial U$. The action of a closed characteristic $\gamma$ on $\partial U$ is defined as $\mathscr{A}(\gamma)=\left|\int_{\gamma} y d x\right|$. The set

$$
\Sigma(U)=\{k \mathscr{A}(\gamma) \mid k=1,2, \ldots ; \gamma \text { is a closed characteristic on } \partial U\}
$$

is called the action spectrum of $U$. Now one would like to associate with $U$ suitable elements of $\Sigma(U)$. Without further assumptions on $U$, however, the set $\Sigma(U)$ may be empty (see $[32 ; 33 ; 35]$ ), and there is no obvious way to achieve (Monotonicity). To salvage this naive idea, Ekeland and Hofer considered for each bounded open subset $U$ of $\mathbb{R}^{2 n}$ the space $\mathscr{F}(U)$ of time-independent Hamiltonian functions $H: \mathbb{R}^{2 n} \rightarrow[0, \infty)$ satisfying

- $H \equiv 0$ on some open neighbourhood of $\bar{U}$, and

- $H(z)=a|z|^{2}$ for $|z|$ large, where $a>\pi, a \notin \mathbb{N} \pi$.

Notice that the circle $S^{1}$ acts on the Hilbert space $E$ by time-shift $x(t) \mapsto$ $x(t+\theta)$ for $\theta \in S^{1}=\mathbb{R} / \mathbb{Z}$. The special form of $H \in \mathscr{F}(U)$ guarantees that for each $k \in \mathbb{N}$ the equivariant minimax value

$$
c_{H, k}:=\inf \left\{\sup _{\gamma \in \xi} \mathscr{A}_{H}(\gamma) \mid \xi \subset E \text { is } S^{1} \text {-equivariant and ind }(\xi) \geq k\right\}
$$

is a critical value of the action functional (2-2). Here, ind $(\xi)$ denotes a suitable Fadell-Rabinowitz index [26; 19] of the intersection $\xi \cap S^{+}$of $\xi$ with the unit sphere $S^{+} \subset E^{+}$. The $k$-th Ekeland-Hofer capacity $c_{k}^{\mathrm{EH}}$ on the symplectic category $O p^{2 n}$ is now defined as

$$
c_{k}^{\mathrm{EH}}(U):=\inf \left\{c_{H, k} \mid H \in \mathscr{F}(U)\right\}
$$

if $U \subset \mathbb{R}^{2 n}$ is bounded and as

$$
c_{k}^{\mathrm{EH}}(U):=\sup \left\{c_{k}^{\mathrm{EH}}(V) \mid V \subset U \text { bounded }\right\}
$$

in general. It turns out that these numbers are indeed symplectic capacities. Moreover, they realize the naive idea of picking out suitable elements of $\Sigma(U)$ for many $U$ : A bounded open subset $U$ of $\mathbb{R}^{2 n}$ is said to be of restricted contact type if its boundary $\partial U$ is smooth and if there exists a vector field $v$ on $\mathbb{R}^{2 n}$ which is transverse to $\partial U$ and whose Lie derivative satisfies $L_{v} \omega_{0}=\omega_{0}$. Examples are bounded star-shaped domains with smooth boundary.

PROPOSITION (EKELAND AND HOFER, 1990). If $U$ is of restricted contact type, then $c_{k}^{\mathrm{EH}}(U) \in \Sigma(U)$ for each $k \in \mathbb{N}$. 
Since the index appearing in the definition of $c_{H, k}$ is monotone, it is immediate from the definition that $c_{1}^{\mathrm{EH}} \leq c_{2}^{\mathrm{EH}} \leq c_{3}^{\mathrm{EH}} \leq \ldots$ form an increasing sequence. Their values on the ball and cylinder are

$$
c_{k}^{\mathrm{EH}}(B)=\left[\frac{k+n-1}{n}\right] \pi \quad \text { and } \quad c_{k}^{\mathrm{EH}}(Z)=k \pi,
$$

where $[x]$ denotes the largest integer $\leq x$. Hence the existence of $c_{1}^{\mathrm{EH}}$ gives an independent proof of Gromov's Nonsqueezing Theorem. Using the capacity $c_{n}^{\mathrm{EH}}$, Ekeland and Hofer [19] also proved the following nonsqueezing result.

TheOREM (EKELAND AND HOFER, 1990). The cube

$$
P=B^{2}(1) \times \ldots \times B^{2}(1) \subset \mathbb{C}^{n}
$$

can be symplectically embedded into the ball $B^{2 n}\left(r^{2}\right)$ if and only if $r^{2} \geq n$.

Other illustrations of the use of Ekeland-Hofer capacities in studying embedding problems for ellipsoids and polydiscs appear in Section 4.

2.3.2. Hofer-Zehnder capacity. (See [48; 49].) Given a symplectic manifold $(M, \omega)$ we consider the class $\mathscr{Y}(M)$ of simple Hamiltonian functions $H: M \rightarrow$ $[0, \infty)$ characterized by the following properties:

- $H=0$ near the (possibly empty) boundary of $M$;

- The critical values of $H$ are 0 and $\max H$.

Such a function is called admissible if the flow $\varphi_{H}^{t}$ of $H$ has no nonconstant periodic orbits with period $T \leq 1$.

The Hofer-Zehnder capacity $c_{\mathrm{HZ}}$ on Symp ${ }^{2 n}$ is defined as

$$
c_{\mathrm{HZ}}(M):=\sup \{\max H \mid H \in \mathscr{Y}(M) \text { is admissible }\}
$$

It measures the symplectic size of $M$ in a dynamical way. Easily constructed examples yield the inequality $c_{\mathrm{HZ}}(B) \geq \pi$. In [48; 49], Hofer and Zehnder applied a minimax technique to the action functional (2-2) to show that $c_{\mathrm{HZ}}(Z) \leq \pi$, so

$$
c_{\mathrm{HZ}}(B)=c_{\mathrm{HZ}}(Z)=\pi,
$$

providing another independent proof of the Nonsqueezing Theorem. Moreover, for every symplectic manifold $(M, \omega)$ the inequality $\pi c_{B}(M) \leq c_{\mathrm{HZ}}(M)$ holds.

The importance of understanding the Hofer-Zehnder capacity comes from the following result proved in $[48 ; 49]$.

THEOREM (HOFER AND ZEHNDER, 1990). Let $H:(M, \omega) \rightarrow \mathbb{R}$ be a proper autonomous Hamiltonian. If $c_{\mathrm{HZ}}(M)<\infty$, then for almost every $c \in H(M)$ the energy level $H^{-1}(c)$ carries a periodic orbit. 
Variants of the Hofer-Zehnder capacity which can be used to detect periodic orbits in a prescribed homotopy class where considered in $[59 ; 88]$.

2.3.3. Displacement energy (See [43; 55].) Next, let us measure the symplectic size of a subset by looking at how much energy is needed to displace it from itself. Fix a symplectic manifold $(M, \omega)$. Given a compactly supported Hamiltonian $H:[0,1] \times M \rightarrow \mathbb{R}$, set

$$
\|H\|:=\int_{0}^{1}\left(\sup _{x \in M} H(t, x)-\inf _{x \in M} H(t, x)\right) d t .
$$

The energy of a compactly supported Hamiltonian diffeomorphism $\varphi$ is

$$
E(\varphi):=\inf \left\{\|H\| \mid \varphi=\varphi_{H}^{1}\right\} .
$$

The displacement energy of a subset $A$ of $M$ is now defined as

$$
e(A, M):=\inf \{E(\varphi) \mid \varphi(A) \cap A=\varnothing\}
$$

if $A$ is compact and as

$$
e(A, M):=\sup \{e(K, M) \mid K \subset A \text { is compact }\}
$$

for a general subset $A$ of $M$.

Now consider the special case $(M, \omega)=\left(\mathbb{R}^{2 n}, \omega_{0}\right)$. Simple explicit examples show $e\left(Z, \mathbb{R}^{2 n}\right) \leq \pi$. In [43], H. Hofer designed a minimax principle for the action functional (2-2) to show that $e\left(B, \mathbb{R}^{2 n}\right) \geq \pi$, so that

$$
e\left(B, \mathbb{R}^{2 n}\right)=e\left(Z, \mathbb{R}^{2 n}\right)=\pi .
$$

It follows that $e\left(\cdot, \mathbb{R}^{2 n}\right)$ is a symplectic capacity on the symplectic category $O p^{2 n}$ of open subsets of $\mathbb{R}^{2 n}$.

One important feature of the displacement energy is the inequality

$$
c_{\mathrm{HZ}}(U) \leq e(U, M)
$$

holding for open subsets of many (and possibly all) symplectic manifolds, including $\left(\mathbb{R}^{2 n}, \omega_{0}\right)$. Indeed, this inequality and the Hofer-Zehnder Theorem imply existence of periodic orbits on almost every energy surface of any Hamiltonian with support in $U$ provided only that $U$ is displaceable in $M$. The proof of this inequality uses the spectral capacities introduced in Section 2.3.4 below.

As an application, consider a closed Lagrangian submanifold $L$ of $\left(\mathbb{R}^{2 n}, \omega_{0}\right)$. Viterbo [98] used an elementary geometric construction to show that

$$
e\left(L, \mathbb{R}^{2 n}\right) \leq C_{n}(\operatorname{vol}(L))^{2 / n}
$$


for an explicit constant $C_{n}$. By a result of Chekanov [12], $e\left(L, \mathbb{R}^{2 n}\right)>0$. Since $e\left(\varphi_{H}(L), \mathbb{R}^{2 n}\right)=e\left(L, \mathbb{R}^{2 n}\right)$ for every Hamiltonian diffeomorphism of $L$, we obtain Viterbo's inequality (1-1).

2.3.4. Spectral capacities. (See $[31 ; 45 ; 49 ; 75 ; 76 ; 77 ; 86 ; 97]$.) For simplicity, we assume again $(M, \omega)=\left(\mathbb{R}^{2 n}, \omega_{0}\right)$. Denote by $\mathscr{H}$ the space of compactly supported Hamiltonian functions $H: S^{1} \times \mathbb{R}^{2 n} \rightarrow \mathbb{R}$. An action selector $\sigma$ selects for each $H \in \mathscr{H}$ the action $\sigma(H)=\mathscr{A}_{H}(\gamma)$ of a "topologically visible" 1periodic orbit $\gamma$ of $\varphi_{H}^{t}$ in a suitable way. Such action selectors were constructed by Viterbo [97], who applied minimax to generating functions, and by Hofer and Zehnder [45; 49], who applied minimax directly to the action functional (2-2). An outline of their constructions can be found in [30].

Given an action selector $\sigma$ for $\left(\mathbb{R}^{2 n}, \omega_{0}\right)$, one defines the spectral capacity $c_{\sigma}$ on the symplectic category $O p^{2 n}$ by

$$
c_{\sigma}(U):=\sup \left\{\sigma(H) \mid H \text { is supported in } S^{1} \times U\right\} .
$$

It follows from the defining properties of an action selector (not given here) that $c_{\mathrm{HZ}}(U) \leq c_{\sigma}(U)$ for any spectral capacity $c_{\sigma}$. Elementary considerations also imply $c_{\sigma}(U) \leq e\left(U, \mathbb{R}^{2 n}\right)$, see [30; 45; 49; 97]. In this way one in particular obtains the important inequality (2-3) for $M=\mathbb{R}^{2 n}$.

Here is another application of action selectors:

THEOREM (VITERBO, 1992). Every nonidentical compactly supported Hamiltonian diffeomorphism of $\left(\mathbb{R}^{2 n}, \omega_{0}\right)$ has infinitely many nontrivial periodic points.

Moreover, the existence of an action selector is an important ingredient in Viterbo's proof of the estimate (1-2) for billiard trajectories.

Using the Floer homology of $(M, \omega)$ filtered by the action functional, an action selector can be constructed for many (and conceivably for all) symplectic manifolds $(M, \omega),[31 ; 75 ; 76 ; 77 ; 86]$. This existence result implies the energycapacity inequality (2-3) for arbitrary open subsets $U$ of such $(M, \omega)$, which has many applications [87].

2.4. Lagrangian capacity. In [16] a capacity is defined on the category of $2 n$-dimensional symplectic manifolds $(M, \omega)$ with $\pi_{1}(M)=\pi_{2}(M)=0$ (with symplectic embeddings as morphisms) as follows. The minimal symplectic area of a Lagrangian submanifold $L \subset M$ is

$$
A_{\min }(L):=\inf \left\{\int_{\sigma} \omega \mid \sigma \in \pi_{2}(M, L), \int_{\sigma} \omega>0\right\} \in[0, \infty] .
$$


The Lagrangian capacity of $(M, \omega)$ is defined as

$c_{L}(M, \omega):=\sup \left\{A_{\min }(L) \mid L \subset M\right.$ is an embedded Lagrangian torus $\}$.

Its values on the ball and cylinder are

$$
c_{L}(B)=\pi / n, \quad c_{L}(Z)=\pi .
$$

As the cube $P=B^{2}(1) \times \ldots \times B^{2}(1)$ contains the standard Clifford torus $T^{n} \subset \mathbb{C}^{n}$, and is contained in the cylinder $Z$, it follows that $c_{L}(P)=\pi$. Together with $c_{L}(B)=\pi / n$ this gives an alternative proof of the nonsqueezing result of Ekeland and Hofer mentioned in Section 2.3.1. There are also applications of the Lagrangian capacity to Arnold's chord conjecture and to Lagrangian (non)embedding results into uniruled symplectic manifolds [16].

\section{General properties and relations between symplectic capacities}

In this section we study general properties of and relations between symplectic capacities. We begin by introducing some more notation. Define the ellipsoids and polydiscs

$$
\begin{aligned}
& E(a):=E\left(a_{1}, \ldots, a_{n}\right):=\left\{z \in \mathbb{C}^{n} \mid \frac{\left|z_{1}\right|^{2}}{a_{1}}+\ldots+\frac{\left|z_{n}\right|^{2}}{a_{n}}<1\right\} \\
& P(a):=P\left(a_{1}, \ldots, a_{n}\right):=B^{2}\left(a_{1}\right) \times \ldots \times B^{2}\left(a_{n}\right)
\end{aligned}
$$

for $0<a_{1} \leq \ldots \leq a_{n} \leq \infty$. Note that in this notation the ball, cube and cylinder are $B=E(1, \ldots, 1), P=P(1, \ldots, 1)$ and $Z=E(1, \infty, \ldots, \infty)=$ $P(1, \infty, \ldots, \infty)$.

Besides $S y m p^{2 n}$ and $O p^{2 n}$, two symplectic categories that will frequently play a role below are

$E l l^{2 n}$ : the category of ellipsoids in $\mathbb{R}^{2 n}$, with symplectic embeddings induced by global symplectomorphisms of $\mathbb{R}^{2 n}$ as morphisms,

Pol $^{2 n}$ : the category of polydiscs in $\mathbb{R}^{2 n}$, with symplectic embeddings induced by global symplectomorphisms of $\mathbb{R}^{2 n}$ as morphisms.

3.1. Generalized symplectic capacities. From the point of view of this work, it is convenient to have a more flexible notion of symplectic capacities, whose axioms were originally designed to explicitly exclude such invariants as the volume. We thus define a generalized symplectic capacity on a symplectic category $\mathscr{C}$ as a covariant functor $c$ from $\mathscr{C}$ to the category $([0, \infty], \leq)$ satisfying only the (Monotonicity) and (Conformality) axioms of Section 2.1. 
Now examples such as the volume capacity on $S y m p^{2 n}$ are included into the discussion. It is defined as

$$
c_{\mathrm{vol}}(M, \omega):=\left(\frac{\operatorname{vol}(M, \omega)}{\operatorname{vol}(B)}\right)^{1 / n},
$$

where $\operatorname{vol}(M, \omega):=\int_{M} \omega^{n} / n$ ! is the symplectic volume. For $n \geq 2$ we have $c_{\mathrm{vol}}(B)=1$ and $c_{\mathrm{vol}}(Z)=\infty$, so $c_{\mathrm{vol}}$ is a normalized generalized capacity but not a capacity. Many more examples appear below.

3.2. Embedding capacities. Let $\mathscr{C}$ be a symplectic category. Every object $(X, \Omega)$ of $\mathscr{C}$ induces two generalized symplectic capacities on $\mathscr{C}$,

$$
\begin{aligned}
& c_{(X, \Omega)}(M, \omega):=\sup \{\alpha>0 \mid(X, \alpha \Omega) \rightarrow(M, \omega)\}, \\
& c^{(X, \Omega)}(M, \omega):=\inf \{\alpha>0 \mid(M, \omega) \rightarrow(X, \alpha \Omega)\},
\end{aligned}
$$

Here the supremum and infimum over the empty set are set to 0 and $\infty$, respectively. Note that

$$
c_{(X, \Omega)}(M, \omega)=\left(c^{(M, \omega)}(X, \Omega)\right)^{-1} .
$$

EXAmPLE 1 . Suppose that $(X, \alpha \Omega) \rightarrow(X, \Omega)$ for some $\alpha>1$. Then

$$
c_{(X, \Omega)}(X, \Omega)=\infty \quad \text { and } \quad c^{(X, \Omega)}(X, \Omega)=0,
$$

so

$$
\begin{aligned}
& c_{(X, \Omega)}(M, \omega)=\left\{\begin{array}{lll}
\infty & \text { if } & (X, \beta \Omega) \rightarrow(M, \omega) \text { for some } \beta>0, \\
0 & \text { if } \quad(X, \beta \Omega) \rightarrow(M, \omega) \text { for no } \beta>0,
\end{array}\right. \\
& c^{(X, \Omega)}(M, \omega)=\left\{\begin{array}{lll}
0 & \text { if } & (M, \omega) \rightarrow(X, \beta \Omega) \text { for some } \beta>0, \\
\infty & \text { if } \quad(M, \omega) \rightarrow(X, \beta \Omega) \text { for no } \beta>0 .
\end{array}\right.
\end{aligned}
$$

The following fact follows directly from the definitions.

FACT 1. Suppose that there exists no morphism $(X, \alpha \Omega) \rightarrow(X, \Omega)$ for any $\alpha>1$. Then $c_{(X, \Omega)}(X, \Omega)=c^{(X, \Omega)}(X, \Omega)=1$, and for every generalized capacity $c$ with $0<c(X, \Omega)<\infty$,

$$
c_{(X, \Omega)}(M, \omega) \leq \frac{c(M, \omega)}{c(X, \Omega)} \leq c^{(X, \Omega)}(M, \omega) \quad \text { for all }(M, \omega) \in \mathscr{C} .
$$

In other words, $c_{(X, \Omega)}\left(\right.$ resp. $\left.c^{(X, \Omega)}\right)$ is the minimal (resp. maximal) generalized capacity $c$ with $c(X, \Omega)=1$. 
Important examples on $S_{y m p}{ }^{2 n}$ arise from the ball $B=B^{2 n}(1)$ and cylinder $Z=Z^{2 n}(1)$. By Gromov's Nonsqueezing Theorem and volume reasons we have for $n \geq 2$ :

$$
c_{B}(Z)=1, \quad c^{Z}(B)=1, \quad c^{B}(Z)=\infty, \quad c_{Z}(B)=0 .
$$

In particular, for every normalized symplectic capacity $c$,

$$
c_{B}(M, \omega) \leq c(M, \omega) \leq c(Z) c^{Z}(M, \omega) \quad \text { for all }(M, \omega) \in \operatorname{Symp}^{2 n} .
$$

Recall that the capacity $c_{B}$ is the Gromov radius defined in Section 2.2. The capacities $c_{B}$ and $c^{Z}$ are not comparable on $O p^{2 n}$ : Example 3 below shows that for every $k \in \mathbb{N}$ there is a bounded star-shaped domain $U_{k}$ of $\mathbb{R}^{2 n}$ such that

$$
c_{B}\left(U_{k}\right) \leq 2^{-k} \quad \text { and } \quad c^{Z}\left(U_{k}\right) \geq \pi k^{2},
$$

see also [42].

We now turn to the question which capacities can be represented as embedding capacities $c_{(X, \Omega)}$ or $c^{(X, \Omega)}$.

EXAMPLE 2. Consider the subcategory $\mathscr{b} \subset O p^{2 n}$ of connected open sets. Then every generalized capacity $c$ on $\mathscr{b}$ can be represented as the capacity $c^{(X, \Omega)}$ of embeddings into a (possibly uncountable) union $(X, \Omega)$ of objects in $\mathscr{C}$.

For this, just define $(X, \Omega)$ as the disjoint union of all $\left(X_{\iota}, \Omega_{\iota}\right)$ in the category $\mathscr{C}$ with $c\left(X_{\iota}, \Omega_{\iota}\right)=0$ or $c\left(X_{\iota}, \Omega_{\iota}\right)=1$.

PROBLEM 1. Which (generalized) capacities can be represented as $c^{(X, \Omega)}$ for a connected symplectic manifold $(X, \Omega)$ ?

PROBLEM 2. Which (generalized) capacities can be represented as the capacity $c_{(X, \Omega)}$ of embeddings from a symplectic manifold $(X, \Omega)$ ?

EXAMPLE 3. Embedding capacities give rise to some curious generalized capacities. For example, consider the capacity $c^{Y}$ of embeddings into the symplectic manifold $Y:=\amalg_{k \in \mathbb{N}} B^{2 n}\left(k^{2}\right)$. It only takes values 0 and $\infty$, with $c^{Y}(M, \omega)=0$ if and only if $(M, \omega)$ embeds symplectically into $Y$; see Example 1 . If $M$ is connected, $\operatorname{vol}(M, \omega)=\infty$ implies $c^{Y}(M, \omega)=\infty$. On the other hand, for every $\varepsilon>0$ there exists an open subset $U \subset \mathbb{R}^{2 n}$, diffeomorphic to a ball, with $\operatorname{vol}(U)<\varepsilon$ and $c^{Y}(U)=\infty$. To see this, consider for $k \in \mathbb{N}$ an open neighbourhood $U_{k}$ of volume $<2^{-k} \varepsilon$ of the linear cone over the Lagrangian torus $\partial B^{2}\left(k^{2}\right) \times \ldots \times \partial B^{2}\left(k^{2}\right)$. The Lagrangian capacity of $U_{k}$ clearly satisfies $c_{L}\left(U_{k}\right) \geq \pi k^{2}$. The open set $U:=\cup_{k \in \mathbb{N}} U_{k}$ satisfies $\operatorname{vol}(U)<\varepsilon$ and $c_{L}(U)=\infty$, hence $U$ does not embed symplectically into any ball. By appropriate choice of the $U_{k}$ we can arrange that $U$ is diffeomorphic to a ball; see [86, Proposition A.3]. 
Special embedding spaces. Given an arbitrary pair of symplectic manifolds $(X, \Omega)$ and $(M, \omega)$, it is a difficult problem to determine or even estimate $c_{(X, \Omega)}(M, \omega)$ and $c^{(X, \Omega)}(M, \omega)$. We thus consider two special cases.

1. Embeddings of skinny ellipsoids. Assume $(M, \omega)$ is an ellipsoid $E(a, \ldots, a, 1)$ with $0<a \leq 1$, and $(X, \Omega)$ is connected and has finite volume. Upper bounds for the function

$$
e^{(X, \Omega)}(a)=c^{(X, \Omega)}(E(a, \ldots, a, 1)), \quad a \in(0,1],
$$

are obtained from symplectic embedding results of ellipsoids into $(X, \Omega)$, and lower bounds are obtained from computing other (generalized) capacities and using Fact 1. In particular, the volume capacity yields

$$
\frac{\left(e^{(X, \Omega)}(a)\right)^{n}}{a^{n-1}} \geq \frac{\operatorname{vol}(B)}{\operatorname{vol}(X, \Omega)} .
$$

The only known general symplectic embedding results for ellipsoids are obtained via multiple symplectic folding. The following result is part of Theorem 3 in [86], which in our setting reads

FACT 2. Assume that $(X, \Omega)$ is a connected $2 n$-dimensional symplectic manifold of finite volume. Then

$$
\lim _{a \rightarrow 0} \frac{\left(e^{(X, \Omega)}(a)\right)^{n}}{a^{n-1}}=\frac{\operatorname{vol}(B)}{\operatorname{vol}(X, \Omega)} .
$$

For a restricted class of symplectic manifolds, Fact 2 can be somewhat improved. The following result is part of Theorem 6.25 of [86].

FACT 3. Assume that $X$ is a bounded domain in $\left(\mathbb{R}^{2 n}, \omega_{0}\right)$ with piecewise smooth boundary or that $(X, \Omega)$ is a compact connected $2 n$-dimensional symplectic manifold. If $n \leq 3$, there exists a constant $C>0$ depending only on $(X, \Omega)$ such that

$$
\frac{\left(e^{(X, \Omega)}(a)\right)^{n}}{a^{n-1}} \leq \frac{\operatorname{vol}(B)}{\operatorname{vol}(X, \Omega)\left(1-C a^{1 / n}\right)} \quad \text { for all } a<\frac{1}{C^{n}} .
$$

These results have their analogues for polydiscs $P(a, \ldots, a, 1)$. The analogue of Fact 3 is known in all dimensions.

2. Packing capacities. Given an object $(X, \Omega)$ of $\mathscr{C}$ and $k \in \mathbb{N}$, we denote by $\bigsqcup_{k}(X, \Omega)$ the disjoint union of $k$ copies of $(X, \Omega)$ and define

$$
c_{(X, \Omega ; k)}(M, \omega):=\sup \left\{\alpha>0 \mid \coprod_{k}(X, \alpha \Omega) \hookrightarrow(M, \omega)\right\} .
$$


If $\operatorname{vol}(X, \Omega)$ is finite, we see as in Fact 1 that

$$
c_{(X, \Omega ; k)}(M, \omega) \leq \frac{1}{c_{\mathrm{vol}}\left(\bigsqcup_{k}(X, \Omega)\right)} c_{\mathrm{vol}}(M, \omega) .
$$

We say that $(M, \omega)$ admits a full $k$-packing by $(X, \Omega)$ if equality holds in (3-3). For $k_{1}, \ldots, k_{n} \in \mathbb{N}$ a full $k_{1} \cdots k_{n}$-packing of $B^{2 n}(1)$ by $E\left(\frac{1}{k_{1}}, \ldots, \frac{1}{k_{n}}\right)$ is given in [94]. E. Ophstein recently showed in [78] that for every closed symplectic manifold $(M, \omega)$ with $[\omega] \in H^{2}(M ; \mathbb{Q})$ there exists a full 1-packing by some ellipsoid. Full $k$-packings by balls and obstructions to full $k$-packings by balls are studied in $[3 ; 4 ; 39 ; 53 ; 63 ; 68 ; 86 ; 94]$.

Assume that also $\operatorname{vol}(M, \omega)$ is finite. Studying the capacity $c_{(X, \Omega ; k)}(M, \omega)$ is equivalent to studying the packing number

$$
p_{(X, \Omega ; k)}(M, \omega)=\sup _{\alpha} \frac{\operatorname{vol}\left(\left(\bigsqcup_{k}(X, \alpha \Omega)\right)\right.}{\operatorname{vol}(M, \omega)}
$$

where the supremum is taken over all $\alpha$ for which $\bigsqcup_{k}(X, \alpha \Omega)$ symplectically embeds into $(M, \omega)$. Clearly, $p_{(X, \Omega ; k)}(M, \omega) \leq 1$, and equality holds if and only if equality holds in (3-3). Results in [68] together with the abovementioned full packings of a ball by ellipsoids from [94] imply

FACT 4. If $X$ is an ellipsoid or a polydisc, then

$$
p_{(X, k)}(M, \omega) \rightarrow 1 \text { as } k \rightarrow \infty
$$

for every symplectic manifold $(M, \omega)$ of finite volume.

Note that if the conclusion of Fact 4 holds for $X$ and $Y$, then it also holds for $X \times Y$.

Problem 3. For which bounded convex subsets $X$ of $\mathbb{R}^{2 n}$ is the conclusion of Fact 4 true?

In [68] and [3; 4], the packing numbers $p_{(X, k)}(M)$ are computed for $X=B^{4}$ and $M=B^{4}$ or $\mathbb{C} P^{2}$. Moreover, the following fact is shown in [3; 4]:

FACT 5. If $X=B^{4}$, then for every closed connected symplectic 4-manifold $(M, \omega)$ with $[\omega] \in H^{2}(M ; \mathbb{Q})$ there exists $k_{0}(M, \omega)$ such that

$$
p_{(X, k)}(M, \omega)=1 \text { for all } k \geq k_{0}(M, \omega) .
$$

Problem 4. For which bounded convex subsets $X$ of $\mathbb{R}^{2 n}$ and which connected symplectic manifolds $(M, \omega)$ of finite volume is the conclusion of Fact 5 true? 
3.3. Operations on capacities. We say that a function $f:[0, \infty]^{n} \rightarrow[0, \infty]$ is homogeneous and monotone if

$$
\begin{aligned}
f\left(\alpha x_{1}, \ldots, \alpha x_{n}\right) & =\alpha f\left(x_{1}, \ldots, x_{n}\right) \quad \text { for all } \alpha>0 \\
f\left(x_{1}, \ldots, x_{i}, \ldots, x_{n}\right) & \leq f\left(x_{1}, \ldots, y_{i}, \ldots, x_{n}\right) \quad \text { for } x_{i} \leq y_{i} .
\end{aligned}
$$

If $f$ is homogeneous and monotone and $c_{1}, \ldots, c_{n}$ are generalized capacities, then $f\left(c_{1}, \ldots, c_{n}\right)$ is again a generalized capacity. If in addition

$$
0<f(1, \ldots, 1)<\infty
$$

and $c_{1}, \ldots, c_{n}$ are capacities, then $f\left(c_{1}, \ldots, c_{n}\right)$ is a capacity. Compositions and pointwise limits of homogeneous monotone functions are again homogeneous and monotone. Examples include $\max \left(x_{1}, \ldots, x_{n}\right), \min \left(x_{1}, \ldots, x_{n}\right)$, and the weighted (arithmetic, geometric, harmonic) means

$$
\lambda_{1} x_{1}+\ldots+\lambda_{n} x_{n}, \quad x_{1}^{\lambda_{1}} \cdots x_{n}^{\lambda_{n}}, \quad \frac{1}{\frac{\lambda_{1}}{x_{1}}+\ldots+\frac{\lambda_{n}}{x_{n}}},
$$

with $\lambda_{1}, \ldots, \lambda_{n} \geq 0, \lambda_{1}+\ldots+\lambda_{n}=1$.

There is also a natural notion of convergence of capacities. We say that a sequence $c_{n}$ of generalized capacities on $\mathscr{C}$ converges pointwise to a generalized capacity $c$ if $c_{n}(M, \omega) \rightarrow c(M, \omega)$ for every $(M, \omega) \in \mathscr{C}$.

These operations yield lots of dependencies between capacities, and it is natural to look for generating systems. In a very general form, this can be formulated as follows.

PROBLEM 5. For a given symplectic category $\mathscr{C}$, find a minimal generating system $\mathscr{G}$ for the (generalized) symplectic capacities on $\mathscr{C}$. This means that every (generalized) symplectic capacity on $\mathscr{C}$ is the pointwise limit of homogeneous monotone functions of elements in $\mathscr{G}$, and no proper subcollection of $\mathscr{G}$ has this property.

This problem is already open for $\mathrm{Ell}^{2 n}$ and $\mathrm{Pol}^{2 n}$. One may also ask for generating systems allowing fewer operations, e.g. only max and min, or only positive linear combinations. We will formulate more specific versions of this problem below. The following simple fact illustrates the use of operations on capacities.

FACT 6. Let $\mathscr{C}$ be a symplectic category containing $B$ (resp. $P$ ). Then every generalized capacity $c$ on $\mathscr{b}$ with $c(B) \neq 0($ resp. $c(P) \neq 0)$ is the pointwise limit of capacities.

Indeed, if $c(B) \neq 0$, then $c$ is the pointwise limit as $k \rightarrow \infty$ of the capacities

$$
c_{k}=\min \left(c, k c_{B}\right),
$$


and likewise with $c(P), c_{P}$ instead of $c(B), c_{B}$.

EXAMPLE 4. (i) The generalized capacity $c \equiv 0$ on $O p^{2 n}$ is not a pointwise limit of capacities, and so the assumption $c(B) \neq 0$ in Fact 6 cannot be omitted.

(ii) The assumption $c(B) \neq 0$ is not always necessary:

(a) Define a generalized capacity $c$ on $O p^{2 n}$ by

$$
c(U)= \begin{cases}0 & \text { if } \operatorname{vol}(U)<\infty, \\ c_{B}(U) & \text { if } \operatorname{vol}(U)=\infty .\end{cases}
$$

Then $c(B)=0$ and $c(Z)=1$, and $c$ is the pointwise limit of the capacities

$$
c_{k}=\max \left(c, \frac{1}{k} c_{B}\right) \text {. }
$$

(b) Define a generalized capacity $c$ on $O p^{2 n}$ by

$$
c(U)= \begin{cases}0 & \text { if } c_{B}(U)<\infty \\ \infty & \text { if } c_{B}(U)=\infty\end{cases}
$$

Then $c(B)=0=c(Z)$ and $c\left(\mathbb{R}^{2 n}\right)=\infty$, and $c=\lim _{k \rightarrow \infty} \frac{1}{k} c_{B}$.

(iii) We do not know whether the generalized capacity $c_{\mathbb{R}^{2 n}}$ on $O p^{2 n}$ is the pointwise limit of capacities.

PROBLem 6. Given a symplectic category $\mathscr{b}$ containing $B$ or $P$ and $Z$, characterize the generalized capacities which are pointwise limits of capacities.

3.4. Continuity. There are several notions of continuity for capacities on open subsets of $\mathbb{R}^{2 n}$, see $[1 ; 18]$. For example, consider a smooth family of hypersurfaces $\left(S_{t}\right)_{-\varepsilon<t<\varepsilon}$ in $\mathbb{R}^{2 n}$, each bounding a compact subset with interior $U_{t}$. Recall that $S_{0}$ is said to be of restricted contact type if there exists a vector field $v$ on $\mathbb{R}^{2 n}$ which is transverse to $S_{0}$ and whose Lie derivative satisfies $L_{v} \omega_{0}=\omega_{0}$. Let $c$ be a capacity on $O p^{2 n}$. As the flow of $v$ is conformally symplectic, the (Conformality) axiom implies the following (see [49, p. 116]):

FACT 7. If $S_{0}$ is of restricted contact type, the function $t \mapsto c\left(U_{t}\right)$ is Lipschitz continuous at 0 .

Fact 7 fails without the hypothesis of restricted contact type. For example, if $S_{0}$ possesses no closed characteristic (such $S_{0}$ exist by $[32 ; 33 ; 35]$ ), then by Theorem 3 in Section 4.2 of [49] the function $t \mapsto c_{\mathrm{HZ}}\left(U_{t}\right)$ is not Lipschitz continuous at 0 . V. Ginzburg [34] presents an example of a smooth family of hypersurfaces $\left(S_{t}\right)$ (albeit not in $\mathbb{R}^{2 n}$ ) for which the function $t \mapsto c_{\mathrm{HZ}}\left(U_{t}\right)$ is not smoother than $1 / 2$-Hölder continuous. These considerations lead to

PROBLEM 7. Are capacities continuous on all smooth families of domains bounded by smooth hypersurfaces? 
3.5. Convex sets. Here we restrict to the subcategory $\operatorname{Conv}^{2 n} \subset O p^{2 n}$ of convex open subsets of $\mathbb{R}^{2 n}$, with embeddings induced by global symplectomorphisms of $\mathbb{R}^{2 n}$ as morphisms. Recall that a subset $U \subset \mathbb{R}^{2 n}$ is star-shaped if $U$ contains a point $p$ such that for every $q \in U$ the straight line between $p$ and $q$ belongs to $U$. In particular, convex domains are star-shaped.

FACT 8. (Extension after Restriction Principle [18]) Assume that $\varphi: U \hookrightarrow \mathbb{R}^{2 n}$ is a symplectic embedding of a bounded star-shaped domain $U \subset \mathbb{R}^{2 n}$. Then for any compact subset $K$ of $U$ there exists a symplectomorphism $\Phi$ of $\mathbb{R}^{2 n}$ such that $\left.\Phi\right|_{K}=\left.\varphi\right|_{K}$.

This principle continues to hold for some, but not all, symplectic embeddings of unbounded star-shaped domains, see [86]. We say that a capacity $c$ defined on a symplectic subcategory of $O p^{2 n}$ has the exhaustion property if

$$
c(U)=\sup \{c(V) \mid V \subset U \text { is bounded }\} .
$$

The capacities introduced in Section 2 all have this property, but the capacity in Example 3 does not. By Fact 8, all statements about capacities defined on a subcategory of $C o n v^{2 n}$ and having the exhaustion property remain true if we allow all symplectic embeddings (not just those coming from global symplectomorphisms of $\mathbb{R}^{2 n}$ ) as morphisms.

FACT 9. Let $U$ and $V$ be objects in Conv ${ }^{2 n}$. Then there exists a morphism $\alpha U \rightarrow V$ for every $\alpha \in(0,1)$ if and only if $c(U) \leq c(V)$ for all generalized capacities $c$ on Conv $^{2 n}$.

Indeed, the necessity of the condition is obvious, and the sufficiency follows by observing that $\alpha U \rightarrow U$ for all $\alpha \in(0,1)$ and $1 \leq c_{U}(U) \leq c_{U}(V)$. What happens for $\alpha=1$ is not well understood, see Section 3.6 for related discussions. The next example illustrates that the conclusion of Fact 9 is wrong without the convexity assumption.

EXAMPLE 5. Consider the open annulus $A=B(4) \backslash B(1)$ in $\mathbb{R}^{2}$. If $\frac{3}{4}<\alpha^{2}<1$, then $\alpha A$ cannot be embedded into $A$ by a global symplectomorphism. Indeed, volume considerations show that any potential such global symplectomorphism would have to map $A$ homotopically nontrivially into itself. This would force the image of the ball $\alpha B(1)$ to cover all of $B(1)$, which is impossible for volume reasons.

Assume now that $c$ is a normalized symplectic capacity on $\operatorname{Conv}^{2 n}$. Using John's ellipsoid, Viterbo [98] noticed that there is a constant $C_{n}$ depending only on $n$ such that

$$
c^{Z}(U) \leq C_{n} c_{B}(U) \text { for all } U \in \operatorname{Conv}^{2 n}
$$


and so, in view of (3-2),

$$
c_{B}(U) \leq c(U) \leq C_{n} c(Z) c_{B}(U) \text { for all } U \in \operatorname{Conv}^{2 n} .
$$

In fact, $C_{n} \leq(2 n)^{2}$ and $C_{n} \leq 2 n$ on centrally symmetric convex sets.

PROBLEM 8. What is the optimal value of the constant $C_{n}$ appearing in (3-5)? In particular, is $C_{n}=1$ ?

Note that $C_{n}=1$ would imply uniqueness of capacities satisfying $c(B)=$ $c(Z)=1$ on $C_{o n v}{ }^{2 n}$. In view of Gromov's Nonsqueezing Theorem, $C_{n}=1$ on $\mathrm{El}^{2 n}$ and $\mathrm{Pol}^{2 n}$. More generally, this equality holds for all convex Reinhardt domains [42]. In particular, for these special classes of convex sets

$$
\pi c_{B}=c_{1}^{\mathrm{EH}}=c_{\mathrm{HZ}}=e\left(\cdot, \mathbb{R}^{2 n}\right)=\pi c^{Z} .
$$

3.6. Recognition. One may ask how complete the information provided by all symplectic capacities is. Consider two objects $(M, \omega)$ and $(X, \Omega)$ of a symplectic category $\mathscr{C}$.

QUESTION 1. Assume $c(M, \omega) \leq c(X, \Omega)$ for all generalized symplectic capacities $c$ on $\mathscr{C}$. Does it follow that $(M, \omega) \hookrightarrow(X, \Omega)$ or even that $(M, \omega) \rightarrow$ $(X, \Omega)$ ?

QUESTION 2. Assume $c(M, \omega)=c(X, \Omega)$ for all generalized symplectic capacities $c$ on $\mathcal{C}$. Does it follow that $(M, \omega)$ is symplectomorphic to $(X, \Omega)$ or even that $(M, \omega) \cong(X, \Omega)$ in the category $\mathscr{C}$ ?

Note that if $(M, \alpha \omega) \rightarrow(M, \omega)$ for all $\alpha \in(0,1)$ then, under the assumptions of Question 1, the argument leading to Fact 9 yields $(M, \alpha \omega) \rightarrow(X, \Omega)$ for all $\alpha \in(0,1)$.

EXAMPLE 6. (i) Set $U=B^{2}(1)$ and $V=B^{2}(1) \backslash\{0\}$. For each $\alpha<1$ there exists a symplectomorphism of $\mathbb{R}^{2}$ with $\varphi(\alpha U) \subset V$, so that monotonicity and conformality imply $c(U)=c(V)$ for all generalized capacities $c$ on $O p^{2}$. Clearly, $U \hookrightarrow V$, but $U \nrightarrow V$, and $U$ and $V$ are not symplectomorphic.

(ii) Set $U=B^{2}(1)$ and let $V=B^{2}(1) \backslash\{(x, y) \mid x \geq 0, y=0\}$ be the slit disc. As is well-known, $U$ and $V$ are symplectomorphic. Fact 8 implies $c(U)=c(V)$ for all generalized capacities $c$ on $O p^{2}$, but clearly $U \nrightarrow V$. In dimensions $2 n \geq 4$ there are bounded convex sets $U$ and $V$ with smooth boundary which are symplectomorphic while $U \nrightarrow V$, see [24].

(iii) Let $U$ and $V$ be ellipsoids in $E l l^{2 n}$. The answer to Question 1 is unknown even for $E l l^{4}$. For $U=E(1,4)$ and $V=B^{4}(2)$ we have $c(U) \leq c(V)$ for all generalized capacities that can presently be computed, but it is unknown whether 
$U \hookrightarrow V$, (see 4.1.2 below). By Fact 10 below the answer to Question 2 is "yes" on $\mathrm{Ell}^{2 n}$.

(iv) Let $U$ and $V$ be polydiscs in $P o l^{2 n}$. Again, the answer to Question 1 is unknown even for $\mathrm{Pol}^{4}$. However, in this dimension the Gromov radius together with the volume capacity determine a polydisc, so that the answer to Question 2 is "yes" on $\mathrm{Pol}^{4}$.

Problem 9. Are two polydiscs in dimension $2 n \geq 6$ with equal generalized symplectic capacities symplectomorphic?

To conclude this section, we mention a specific example in which $c(U)=c(V)$ for all known (but possibly not for all) generalized symplectic capacities.

EXAMPLE 7. Consider the subsets

$$
U=E(2,6) \times E(3,3,6) \quad \text { and } \quad V=E(2,6,6) \times E(3,3)
$$

of $\mathbb{R}^{10}$. Then $c(U)=c(V)$ whenever $c(B)=c(Z)$ by the Nonsqueezing Theorem, the volumes agree, and $c_{k}^{\mathrm{EH}}(U)=c_{k}^{E H}(V)$ for all $k$ by the product formula (3-8). It is unknown whether $U \hookrightarrow V$ or $V \hookrightarrow U$ or $U \rightarrow V$. Symplectic homology as constructed in [28; 93] does not help in these problems because a computation based on [29] shows that all symplectic homologies of $U$ and $V$ agree.

3.7. Hamiltonian representability. Consider a bounded domain $U \subset \mathbb{R}^{2 n}$ with smooth boundary of restricted contact type (see Section 2.3.1 for the definition). As in 2.3.1 we consider the action spectrum

$$
\Sigma(U)=\left\{k\left|\int_{\gamma} y d x\right| \mid k=1,2, \ldots ; \gamma \text { is a closed characteristic on } \partial U\right\}
$$

of $U$. This set is nowhere dense in $\mathbb{R}$ (compare [49, Section 5.2]), and it is easy to see that $\Sigma(U)$ is closed and $0 \notin \Sigma(U)$. For many capacities $c$ constructed via Hamiltonian systems, such as Ekeland-Hofer capacities $c_{k}^{\mathrm{EH}}$ and spectral capacities $c_{\sigma}$, one has $c(U) \in \Sigma(U)$, see [19; 41]. Moreover,

$$
c_{\mathrm{HZ}}(U)=c_{1}^{\mathrm{EH}}(U)=\min (\Sigma(U)) \quad \text { if } U \text { is convex. }
$$

One might therefore be tempted to ask

QUESTION 3. Is it true that $\pi c(U) \in \Sigma(U)$ for every normalized symplectic capacity $c$ on $\mathrm{Op}^{2 n}$ and every domain $U$ with boundary of restricted contact type?

The following example due to D. Hermann [42] shows that the answer to Question 3 is "no". 
EXAMPLE 8. Choose any $U$ with boundary of restricted contact type such that

$$
c_{B}(U)<c^{Z}(U)
$$

Examples are bounded star-shaped domains $U$ with smooth boundary which contain the Lagrangian torus $S^{1} \times \ldots \times S^{1}$ but have small volume: According to [91], $c^{Z}(U) \geq 1$, while $c_{B}(U)$ is as small as we like. Now notice that for each $t \in[0,1]$,

$$
c_{t}=(1-t) c_{B}+t c^{Z}
$$

is a normalized symplectic capacity on $O p^{2 n}$. By (3-7), the interval

$$
\left\{c_{t}(U) \mid t \in[0,1]\right\}=\left[c_{B}(U), c^{Z}(U)\right]
$$

has positive measure and hence cannot lie in the nowhere dense set $\Sigma(U)$.

D. Hermann also pointed out that the argument in Example 8 together with (3-6) implies that the question " $C_{n}=1$ ?" posed in Problem 8 is equivalent to Question 3 for convex sets.

3.8. Products. Consider a family of symplectic categories $\mathscr{b}^{2 n}$ in all dimensions $2 n$ such that

$$
(M, \omega) \in \mathscr{C}^{2 m}, \quad(N, \sigma) \in \mathscr{C}^{2 n} \Longrightarrow(M \times N, \omega \oplus \sigma) \in \mathscr{C}^{2(m+n)} .
$$

We say that a collection $c: \amalg_{n=1}^{\infty} \mathscr{b}^{2 n} \rightarrow[0, \infty]$ of generalized capacities has the product property if

$$
c(M \times N, \omega \oplus \sigma)=\min \{c(M, \omega), c(N, \sigma)\}
$$

for all $(M, \omega) \in \mathscr{C}^{2 m},(N, \sigma) \in \mathscr{C}^{2 n}$. If $\mathbb{R}^{2} \in \mathscr{C}^{2}$ and $c\left(\mathbb{R}^{2}\right)=\infty$, the product property implies the stability property

$$
c\left(M \times \mathbb{R}^{2}, \omega \oplus \omega_{0}\right)=c(M, \omega)
$$

for all $(M, \omega) \in \mathscr{C}^{2 m}$.

EXAMPLE 9. (i) Let $\Sigma_{g}$ be a closed surface of genus $g$ endowed with an area form $\omega$. Then

$$
c_{B}\left(\Sigma_{g} \times \mathbb{R}^{2}, \omega \oplus \omega_{0}\right)= \begin{cases}c_{B}\left(\Sigma_{g}, \omega\right)=\frac{1}{\pi} \omega\left(\Sigma_{g}\right) & \text { if } g=0, \\ \infty & \text { if } g \geq 1 .\end{cases}
$$

While the result for $g=0$ follows from Gromov's Nonsqueezing Theorem, the result for $g \geq 1$ belongs to Polterovich [69, Exercise 12.4] and Jiang [52]. Since $c_{B}$ is the smallest normalized symplectic capacity on $S y m p^{2 n}$, we find that no collection $c$ of symplectic capacities defined on the family $\bigsqcup_{n=1}^{\infty} S y m p^{2 n}$ with $c\left(\Sigma_{g}, \omega\right)<\infty$ for some $g \geq 1$ has the product or stability property. 
(ii) On the family of polydiscs $\bigsqcup_{n=1}^{\infty} P o l^{2 n}$, the Gromov radius, the Lagrangian capacity and the unnormalized Ekeland-Hofer capacities $c_{k}^{\mathrm{EH}}$ all have the product property (see Section 4.2). The volume capacity is not stable.

(iii) Let $U \in O p^{2 m}$ and $V \in O p^{2 n}$ have smooth boundary of restricted contact type (see Section 3.4 for the definition). The formula

$$
c_{k}^{\mathrm{EH}}(U \times V)=\min _{i+j=k}\left(c_{i}^{\mathrm{EH}}(U)+c_{j}^{\mathrm{EH}}(V)\right),
$$

in which we set $c_{0}^{\mathrm{EH}} \equiv 0$, was conjectured by Floer and Hofer [95] and has been proved by Chekanov [13] as an application of his equivariant Floer homology. Consider the collection of sets $U_{1} \times \ldots \times U_{l}$, where each $U_{i} \in O p^{2 n_{i}}$ has smooth boundary of restricted contact type, and $\sum_{i=1}^{l} n_{i}=n$. We denote by $R C T^{2 n}$ the corresponding category with symplectic embeddings induced by global symplectomorphisms of $\mathbb{R}^{2 n}$ as morphisms. If $v_{i}$ are vector fields on $\mathbb{R}^{2 n_{i}}$ with $L_{v_{i}} \omega_{0}=\omega_{0}$, then $L_{v_{1}+\ldots+v_{l}} \omega_{0}=\omega_{0}$ on $\mathbb{R}^{2 n}$. Elements of $R C T^{2 n}$ can therefore be exhausted by elements of $R C T^{2 n}$ with smooth boundary of restricted contact type. This and the exhaustion property (3-4) of the $c_{k}^{\mathrm{EH}}$ shows that (3-8) holds for all $U \in R C T^{2 m}$ and $V \in R C T^{2 n}$, implying in particular that Ekeland-Hofer capacities are stable on $R C T:=\bigsqcup_{n=1}^{\infty} R C T^{2 n}$. Moreover, (3-8) yields that

$$
c_{k}^{\mathrm{EH}}(U \times V) \leq \min \left(c_{k}^{\mathrm{EH}}(U), c_{k}^{\mathrm{EH}}(V)\right),
$$

and it shows that $c_{1}^{\mathrm{EH}}$ on $R C T$ has the product property. Using (3-8) together with an induction over the number of factors and $c_{2}^{\mathrm{EH}}\left(E\left(a_{1}, \ldots, a_{n}\right)\right) \leq 2 a_{1}$ we also see that $c_{2}^{\mathrm{EH}}$ has the product property on products of ellipsoids. For $k \geq 3$, however, the Ekeland-Hofer capacities $c_{k}^{\mathrm{EH}}$ on $R C T$ do not have the product property. As an example, for $U=B^{4}(4)$ and $V=E(3,8)$ we have

$$
c_{3}^{\mathrm{EH}}(U \times V)=7<8=\min \left(c_{3}^{\mathrm{EH}}(U), c_{3}^{\mathrm{EH}}(V)\right) .
$$

PROBLEM 10. Characterize the collections of (generalized) capacities on polydiscs that have the product (resp. stability) property.

Next consider a collection $c$ of generalized capacities on open subsets $O p^{2 n}$. In general, it will not be stable. However, we can stabilize $c$ to obtain stable generalized capacities $c^{ \pm}: \coprod_{n=1}^{\infty} O p^{2 n} \rightarrow[0, \infty]$,

$$
c^{+}(U):=\limsup _{k \rightarrow \infty} c\left(U \times \mathbb{R}^{2 k}\right), \quad c^{-}(U):=\liminf _{k \rightarrow \infty} c\left(U \times \mathbb{R}^{2 k}\right) .
$$

Notice that $c(U)=c^{+}(U)=c^{-}(U)$ for all $U \in \bigsqcup_{n=1}^{\infty} O p^{2 n}$ if and only if $c$ is stable. If $c$ consists of capacities and there exist constants $a, A>0$ such that

$$
a \leq c\left(B^{2 n}(1)\right) \leq c\left(Z^{2 n}(1)\right) \leq A \quad \text { for all } n \in \mathbb{N},
$$


then $c^{ \pm}$are collections of capacities. Thus there exist plenty of stable capacities on $O p^{2 n}$. However, we have

Problem 11. Decide stability of specific collections of capacities on Conv ${ }^{2 n}$ or $\mathrm{Op}^{2 n}$, e.g.: Gromov radius, Ekeland-Hofer capacity, Lagrangian capacity, and the embedding capacity $c_{P}$ of the unit cube.

PROBLEM 12. Does there exist a collection of capacities on $\bigsqcup_{n=1}^{\infty}$ Conv ${ }^{2 n}$ or $\bigsqcup_{n=1}^{\infty} O p^{2 n}$ with the product property?

3.9. Higher order capacities? Following [44], we briefly discuss the concept of higher order capacities. Consider a symplectic category $\mathscr{b} \subset S y m p^{2 n}$ containing $E l l^{2 n}$ and fix $d \in\{1, \ldots, n\}$. A symplectic $d$-capacity on $\mathscr{b}$ is a generalized capacity satisfying

$(d$-Nontriviality): $0<c(B)$ and

$$
\left\{\begin{array}{l}
c\left(B^{2 d}(1) \times \mathbb{R}^{2(n-d)}\right)<\infty, \\
c\left(B^{2(d-1)}(1) \times \mathbb{R}^{2(n-d+1)}\right)=\infty .
\end{array}\right.
$$

For $d=1$ we recover the definition of a symplectic capacity, and for $d=n$ the volume capacity $c_{\mathrm{vol}}$ is a symplectic $n$-capacity.

PROBLEM 13. Does there exist a symplectic d-capacity on a symplectic category $\mathscr{C}$ containing $\mathrm{Ell}^{2 n}$ for some $d \in\{2, \ldots, n-1\}$ ?

Problem 13 on $S_{y m p}{ }^{2 n}$ is equivalent to the following symplectic embedding problem.

Problem 14. Does there exist a symplectic embedding

$$
B^{2(d-1)}(1) \times \mathbb{R}^{2(n-d+1)} \hookrightarrow B^{2 d}(R) \times \mathbb{R}^{2(n-d)}
$$

for some $R<\infty$ and $d \in\{2, \ldots, n-1\}$ ?

Indeed, the existence of such an embedding would imply that no symplectic $d$ capacity can exist on $S y m p^{2 n}$. Conversely, if no such embedding exists, then the embedding capacity $c^{Z_{2 d}}$ into $Z_{2 d}=B^{2 d}(1) \times \mathbb{R}^{2(n-d)}$ would be an example of a $d$-capacity on $S y m p^{2 n}$. The Ekeland-Hofer capacity $c_{d}^{\mathrm{EH}}$ shows that $R$ is at least 2 if a symplectic embedding (3-9) exists. The known symplectic embedding techniques are not designed to effectively use the unbounded factor of the target space in (3-9). E.g., multiple symplectic folding only shows that there exists a function $f:[1, \infty) \rightarrow \mathbb{R}$ with $f(a)<\sqrt{2 a}+2$ such that for each $a \geq 1$ there exists a symplectic embedding

$$
B^{2}(1) \times B^{2}(a) \times \mathbb{R}^{2} \hookrightarrow B^{4}(f(a)) \times \mathbb{R}^{2}
$$

of the form $\varphi \times i d_{2}$, see [86, Section 4.3.2]. 


\section{Ellipsoids and polydiscs}

In this section we investigate generalized capacities on the categories of ellipsoids $\mathrm{Ell}^{2 n}$ and polydiscs $\mathrm{Pol}^{2 n}$ in more detail. All (generalized) capacities $c$ in this section are defined on some symplectic subcategory of $O p^{2 n}$ containing at least one of the above categories and are assumed to have the exhaustion property (3-4).

\subsection{Ellipsoids.}

4.1.1. Arbitrary dimension. We first describe the values of the capacities introduced in Section 2 on ellipsoids.

The values of the Gromov radius $c_{B}$ on ellipsoids are

$$
c_{B}\left(E\left(a_{1}, \ldots, a_{n}\right)\right)=\min \left\{a_{1}, \ldots, a_{n}\right\} .
$$

More generally, monotonicity implies that this formula holds for all symplectic capacities $c$ on $O p^{2 n}$ with $c(B)=c(Z)=1$ and hence also for $\frac{1}{\pi} c_{1}^{\mathrm{EH}}, \frac{1}{\pi} c_{\mathrm{HZ}}$, $\frac{1}{\pi} e\left(\cdot, \mathbb{R}^{2 n}\right)$ and $c^{Z}$.

The values of the Ekeland-Hofer capacities on the ellipsoid $E\left(a_{1}, \ldots, a_{n}\right)$ can be described as follows [19]. Write the numbers $m a_{i} \pi, m \in \mathbb{N}, 1 \leq i \leq n$, in increasing order as $d_{1} \leq d_{2} \leq \ldots$, with repetitions if a number occurs several times. Then

$$
c_{k}^{\mathrm{EH}}\left(E\left(a_{1}, \ldots, a_{n}\right)\right)=d_{k} .
$$

The values of the Lagrangian capacity on ellipsoids are presently not known. In [16], Cieliebak and Mohnke make the following conjecture:

\section{CONJeCTURe 1.}

$$
c_{L}\left(E\left(a_{1}, \ldots, a_{n}\right)\right)=\frac{\pi}{1 / a_{1}+\ldots+1 / a_{n}} .
$$

Since $\operatorname{vol}\left(E\left(a_{1}, \ldots, a_{n}\right)\right)=a_{1} \cdots a_{n} \operatorname{vol}(B)$, the values of the volume capacity on ellipsoids are

$$
c_{\mathrm{vol}}\left(E\left(a_{1}, \ldots, a_{n}\right)\right)=\left(a_{1} \cdots a_{n}\right)^{1 / n} .
$$

In view of conformality and the exhaustion property, a (generalized) capacity on $E l l^{2 n}$ is determined by its values on the ellipsoids $E\left(a_{1}, \ldots, a_{n}\right)$ with $0<$ $a_{1} \leq \ldots \leq a_{n}=1$. So we can view each (generalized) capacity $c$ on ellipsoids as a function

$$
c\left(a_{1}, \ldots, a_{n-1}\right):=c\left(E\left(a_{1}, \ldots, a_{n-1}, 1\right)\right)
$$

on the set $\left\{0<a_{1} \leq \ldots \leq a_{n-1} \leq 1\right\}$. By Fact 7, this function is continuous. This identification with functions yields a notion of uniform convergence for capacities on $\mathrm{Ell}^{2 n}$. 
For what follows, it is useful to have normalized versions of the EkelandHofer capacities, so in dimension $2 n$ we define

$$
\bar{c}_{k}:=\frac{c_{k}^{\mathrm{EH}}}{\left[\frac{k+n-1}{n}\right] \pi} .
$$

Proposition 1. As $k \rightarrow \infty$, for every $n \geq 2$ the normalized Ekeland-Hofer capacities $\bar{c}_{k}$ converge uniformly on Ell ${ }^{2 n}$ to the normalized symplectic capacity $c_{\infty}$ given by

$$
c_{\infty}\left(E\left(a_{1}, \ldots, a_{n}\right)\right)=\frac{n}{1 / a_{1}+\ldots+1 / a_{n}} .
$$

REMARK. Note that Conjecture 1 asserts that $c_{\infty}$ agrees with the normalized Lagrangian capacity $\bar{c}_{L}=n c_{L} / \pi$ on $E l l^{2 n}$.

Proof of Proposition 1. Fix $\varepsilon>0$. We need to show that $\left|\bar{c}_{k}(a)-c_{\infty}(a)\right| \leq$ $\varepsilon$ for every vector $a=\left(a_{1}, \ldots, a_{n}\right)$ with $0<a_{1} \leq a_{2} \leq \ldots \leq a_{n}=1$ and all sufficiently large $k$. Abbreviate $\delta=\varepsilon / n$.

Case 1. $a_{1} \leq \delta$. Then

$$
c_{k}^{\mathrm{EH}}(a) \leq k \delta \pi, \quad \bar{c}_{k}(a) \leq n \delta, \quad c_{\infty}(a) \leq n \delta
$$

from which we conclude $\left|\bar{c}_{k}(a)-c_{\infty}(a)\right| \leq n \delta=\varepsilon$ for all $k \geq 1$.

Case 2. $a_{1}>\delta$. Let $k \geq 2 \frac{n-1}{\delta}+2$. For the unique integer $l$ with

$$
\pi l a_{n} \leq c_{k}^{\mathrm{EH}}(a)<\pi(l+1) a_{n}
$$

we then have $l \geq 2$. In the increasing sequence of the numbers $m a_{i}(m \in \mathbb{N}$, $1 \leq i \leq n)$, the first $\left[l a_{n} / a_{i}\right]$ multiples of $a_{i}$ occur no later than $l a_{n}$. By the description of the Ekeland-Hofer capacities on ellipsoids given above, this yields the estimates

$$
\frac{(l-1) a_{n}}{a_{1}}+\ldots+\frac{(l-1) a_{n}}{a_{n}} \leq k \leq \frac{(l+1) a_{n}}{a_{1}}+\ldots+\frac{(l+1) a_{n}}{a_{n}} .
$$

With $\gamma:=a_{n} / a_{1}+\ldots+a_{n} / a_{n}$ this becomes

$$
(l-1) \gamma \leq k \leq(l+1) \gamma .
$$

Using $\gamma \geq n$, we derive the inequalities

$$
\begin{aligned}
& {\left[\frac{k+n-1}{n}\right] \leq \frac{k}{n}+1 \leq \frac{(l+1) \gamma+n}{n} \leq \frac{(l+2) \gamma}{n},} \\
& {\left[\frac{k+n-1}{n}\right] \geq \frac{k}{n} \geq \frac{(l-1) \gamma}{n} .}
\end{aligned}
$$


With the definition of $\bar{c}_{k}$ and the estimate above for $c_{k}^{\mathrm{EH}}$, we find

$$
\frac{n l a_{n}}{(l+2) \gamma} \leq \bar{c}_{k}(a)=\frac{c_{k}^{\mathrm{EH}}(a)}{\left[\frac{k+n-1}{n}\right] \pi} \leq \frac{n(l+1) a_{n}}{(l-1) \gamma} .
$$

Since $c_{\infty}(a)=n a_{n} / \gamma$, this becomes

$$
\frac{l}{l+2} c_{\infty}(a) \leq \bar{c}_{k}(a) \leq \frac{l+1}{l-1} c_{\infty}(a),
$$

which in turn implies

$$
\left|\bar{c}_{k}(a)-c_{\infty}(a)\right| \leq \frac{2 c_{\infty}(a)}{l-1}
$$

Since $a_{1}>\delta$ we have

$$
\gamma \leq \frac{n}{\delta}, \quad l+1 \geq \frac{k}{\gamma} \geq \frac{k \delta}{n},
$$

from which we conclude

$$
\left|\bar{c}_{k}(a)-c_{\infty}(a)\right| \leq \frac{2}{l-1} \leq \frac{2 n}{k \delta-2 n} \leq \varepsilon
$$

for $k$ sufficiently large.

We turn to the question whether Ekeland-Hofer capacities generate the space of all capacities on ellipsoids by suitable operations. First note some easy facts.

FACT 10. An ellipsoid $E \subset \mathbb{R}^{2 n}$ is uniquely determined by its Ekeland-Hofer capacities $c_{1}^{\mathrm{EH}}(E), c_{2}^{\mathrm{EH}}(E), \ldots$.

Indeed, if $E(a)$ and $E(b)$ are two ellipsoids with $a_{i}=b_{i}$ for $i<k$ and $a_{k}<b_{k}$, then the multiplicity of $a_{k}$ in the sequence of Ekeland-Hofer capacities is one higher for $E(a)$ than for $E(b)$, so not all Ekeland-Hofer capacities agree.

FACT 11. For every $k \in \mathbb{N}$ there exist ellipsoids $E$ and $E^{\prime}$ with $c_{i}^{\mathrm{EH}}(E)=$ $c_{i}^{\mathrm{EH}}\left(E^{\prime}\right)$ for $i<k$ and $c_{k}^{\mathrm{EH}}(E) \neq c_{k}^{\mathrm{EH}}\left(E^{\prime}\right)$.

For example, we can take $E=E(a)$ and $E^{\prime}=E(b)$ with $a_{1}=b_{1}=1$, $a_{2}=k-1 / 2, b_{2}=k+1 / 2$, and $a_{i}=b_{i}=2 k$ for $i \geq 3$. So formally, every generalized capacity on ellipsoids is a function of the Ekeland-Hofer capacities, and the Ekeland-Hofer capacities are functionally independent. However, Ekeland-Hofer capacities do not form a generating system for symplectic capacities on $\mathrm{Ell}^{2 n}$ (see Example 10 below), and on bounded ellipsoids each finite set of Ekeland-Hofer capacities is determined by the (infinitely many) other Ekeland-Hofer capacities: 
LEMMA 1. Let $d_{1} \leq d_{2} \leq \ldots$ be an increasing sequence of real numbers obtained from the sequence $c_{1}^{\mathrm{EH}}(E) \leq c_{2}^{\mathrm{EH}}(E) \leq \ldots$ of Ekeland-Hofer capacities of a bounded ellipsoid $E \in E l l^{2 n}$ by removing at most $N_{0}$ numbers. Then $E$ can be recovered uniquely.

ProOF. We first consider the special case in which $E=E\left(a_{1}, \ldots, a_{n}\right)$ is such that $a_{i} / a_{j} \in \mathbb{Q}$ for all $i, j$. In this case, the sequence $d_{1} \leq d_{2} \leq \ldots$ contains infinitely many blocks of $n$ consecutive equal numbers. We traverse the sequence until we have found $N_{0}+1$ such blocks, for each block $d_{k}=d_{k+1}=\ldots=d_{k+n-1}$ recording the number $g_{k}:=d_{k+n}-d_{k}$. The minimum of the $g_{k}$ for the $N_{0}+1$ first blocks equals $a_{1}$. After deleting each occurring positive integer multiple of $a_{1}$ once from the sequence $d_{1} \leq d_{2} \leq \ldots$, we can repeat the same procedure to determine $a_{2}$, and so on.

In general, we do not know whether or not $a_{i} / a_{j} \in \mathbb{Q}$ for all $i, j$. To reduce to the previous case, we split the sequence $d_{1} \leq d_{2} \leq \ldots$ into (at most $n$ ) subsequences of numbers with rational quotients. More precisely we traverse the sequence, grouping the $d_{i}$ into increasing subsequences $s_{1}, s_{2}, \ldots$, where each new number is added to the first subsequence $s_{j}$ whose members are rational multiples of it. Furthermore, in this process we record for each sequence $s_{j}$ the maximal length $l_{j}$ of a block of consecutive equal numbers seen so far. We stop when

(i) the sum of the $l_{j}$ equals $n$, and

(ii) each subsequence $s_{j}$ contains at least $N_{0}+1$ blocks of $l_{j}$ consecutive equal numbers.

Now the previously described procedure in the case that $a_{i} / a_{j} \in \mathbb{Q}$ for all $i, j$ can be applied for each subsequence $s_{j}$ separately, where $l_{j}$ replaces $n$ in the above argument.

REMARK. If the volume of $E$ is known, one does not need to know $N_{0}$ in Fact 1. The proof of this is left to the interested reader.

The set of Ekeland-Hofer capacities does not form a generating system for symplectic capacities on $E l l^{2 n}$. Indeed, the volume capacity $c_{\mathrm{vol}}$ is not the pointwise limit of homogeneous monotone functions of Ekeland-Hofer capacities:

EXAmPle 10. Consider the ellipsoids $E=E\left(1, \ldots, 1,3^{n}+1\right)$ and $F=$ $E(3, \ldots, 3)$ in $E l l^{2 n}$. As is easy to see,

$$
c_{k}^{\mathrm{EH}}(E)<c_{k}^{\mathrm{EH}}(F) \quad \text { for all } k
$$


Assume that $f_{i}$ is a sequence of homogeneous monotone functions of EkelandHofer capacities which converge pointwise to $c_{\mathrm{vol}}$. By (4-1) and the monotonicity of the $f_{i}$ we would find that $c_{\mathrm{vol}}(E) \leq c_{\mathrm{vol}}(F)$. This is not true.

Problem 15. Do the Ekeland-Hofer capacities together with the volume capacity form a generating system for symplectic capacities on $\mathrm{Ell}^{2 n}$ ?

If the answer to this problem is "yes", this is a very difficult problem as Lemma 2 below illustrates.

4.1.2. Ellipsoids in dimension 4. A generalized capacity on ellipsoids in dimension 4 is represented by a function $c(a):=c(E(a, 1))$ of a single real variable $0<a \leq 1$. This function has the following two properties.

(i) The function $c(a)$ is nondecreasing.

(ii) The function $c(a) / a$ is nonincreasing.

The first property follows directly from the (Monotonicity) axiom. The second property follows from (Monotonicity) and (Conformality): For $a \leq b, E(b, 1) \subset$ $E\left(\frac{b}{a} a, \frac{b}{a}\right)$, hence $c(b) \leq \frac{b}{a} c(a)$. Note that property (ii) is equivalent to the estimate

$$
\frac{c(b)-c(a)}{b-a} \leq \frac{c(a)}{a}
$$

for $0<a<b$, so the function $c(a)$ is Lipschitz continuous at all $a>0$. We will restrict our attention to normalized (generalized) capacities, so the function $c$ also satisfies

(iii) $c(1)=1$.

An ellipsoid $E\left(a_{1}, \ldots, a_{n}\right)$ embeds into $E\left(b_{1}, \ldots, b_{n}\right)$ by a linear symplectic embedding only if $a_{i} \leq b_{i}$ for all $i$, see [49]. Hence for normalized capacities on the category $\mathrm{LinEll}^{4}$ of ellipsoids with linear embeddings as morphisms, properties (i), (ii) and (iii) are the only restrictions on the function $c(a)$. On $E l l^{4}$, nonlinear symplectic embeddings ("folding") yield additional constraints which are still not completely known; see [86] for the presently known results.

By Fact 1 , the embedding capacities $c_{B}$ and $c^{B}$ are the smallest, resp. largest, normalized capacities on ellipsoids. By Gromov's Nonsqueezing Theorem, $c_{B}(a)=\bar{c}_{1}(a)=a$. The function $c^{B}(a)$ is not completely known. Fact 1 applied to $\bar{c}_{2}$ yields

$$
c^{B}(a)=1 \text { if } a \in\left[\frac{1}{2}, 1\right] \quad \text { and } \quad c^{B}(a) \geq 2 a \text { if } a \in\left(0, \frac{1}{2}\right],
$$


and Fact 1 applied to $c_{\mathrm{vol}}$ yields $c^{B}(a) \geq \sqrt{a}$. Folding constructions provide upper bounds for $c^{B}(a)$. Lagrangian folding [94] yields $c^{B}(a) \leq l(a)$ where

$$
l(a)=\left\{\begin{array}{cl}
(k+1) a & \text { for } \frac{1}{k(k+1)} \leq a \leq \frac{1}{(k-1)(k+1)} \\
\frac{1}{k} & \text { for } \frac{1}{k(k+2)} \leq a \leq \frac{1}{k(k+1)}
\end{array}\right.
$$

and multiple symplectic folding [86] yields $c^{B}(a) \leq s(a)$ where the function $s(a)$ is as shown in Figure 1. While symplectically folding once yields $c^{B}(a) \leq$ $a+1 / 2$ for $a \in(0,1 / 2]$, the function $s(a)$ is obtained by symplectically folding "infinitely many times", and it is known that

$$
\liminf _{\varepsilon \rightarrow 0^{+}} \frac{c^{B}\left(\frac{1}{2}\right)-c^{B}\left(\frac{1}{2}-\varepsilon\right)}{\varepsilon} \geq \frac{8}{7} .
$$

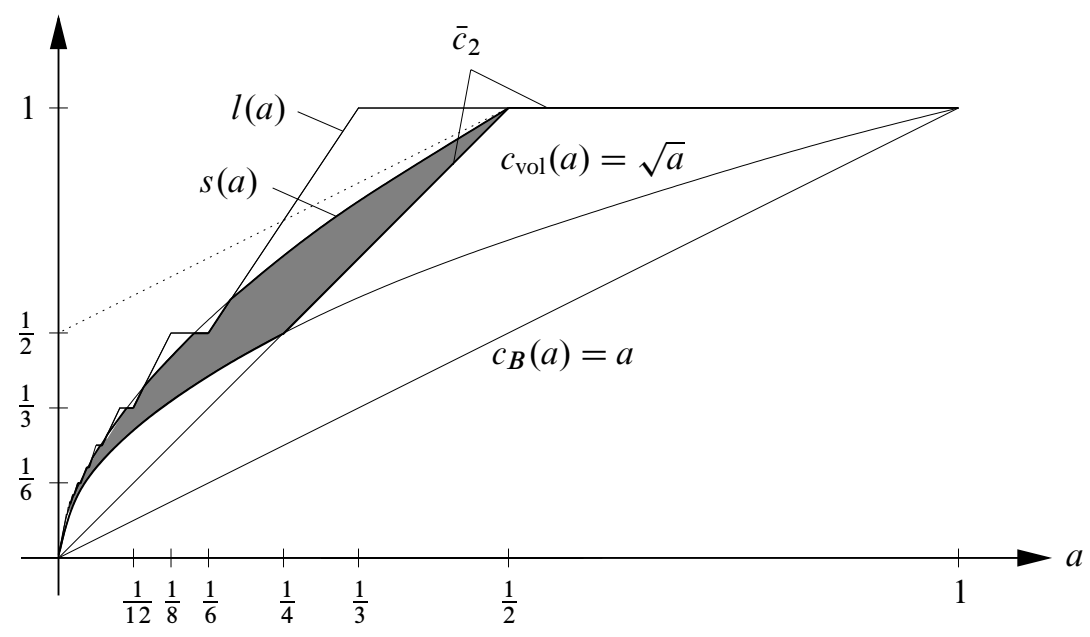

Figure 1. Lower and upper bounds for $c^{B}(a)$.

Let us come back to Problem 15.

LEMMA 2. If the Ekeland-Hofer capacities and the volume capacity form a generating system for symplectic capacities on $\mathrm{Ell}^{2 n}$, then $c^{B}\left(\frac{1}{4}\right)=\frac{1}{2}$.

We recall that $c^{B}\left(\frac{1}{4}\right)=\frac{1}{2}$ means that the ellipsoid $E(1,4)$ symplectically embeds into $B^{4}(2+\varepsilon)$ for every $\varepsilon>0$.

Proof of Lemma 2. We can assume that all capacities are normalized. By assumption, there exists a sequence $f_{i}$ of homogeneous and monotone functions in the $\bar{c}_{k}$ and in $c_{\mathrm{vol}}$ forming normalized capacities which pointwise converge to 
$c^{B}$. As is easy to see, $\bar{c}_{k}\left(E\left(\frac{1}{4}, 1\right)\right) \leq \bar{c}_{k}\left(B^{4}\left(\frac{1}{2}\right)\right)$ for all $k$, and $c_{\mathrm{vol}}\left(E\left(\frac{1}{4}, 1\right)\right)=$ $c_{\mathrm{vol}}\left(B^{4}\left(\frac{1}{2}\right)\right)$. Since the $f_{i}$ are monotone and converge in particular at $E\left(\frac{1}{4}, 1\right)$ and $B^{4}\left(\frac{1}{2}\right)$ to $c^{B}$, we conclude that $c^{B}\left(\frac{1}{4}\right)=c^{B}\left(E\left(\frac{1}{4}, 1\right)\right) \leq c^{B}\left(B^{4}\left(\frac{1}{2}\right)\right)=\frac{1}{2}$, which proves Lemma 2.

In view of Lemma 2, the following problem is a special case of Problem 15.

Problem 16. Is it true that $c^{B}\left(\frac{1}{4}\right)=\frac{1}{2}$ ?

The best upper bound for $c^{B}\left(\frac{1}{4}\right)$ presently known is $s\left(\frac{1}{4}\right) \approx 0.6729$. Answering Problem 16 in the affirmative means to construct for each $\varepsilon>0$ a symplectic embedding $E\left(\frac{1}{4}, 1\right) \rightarrow B^{4}\left(\frac{1}{2}+\varepsilon\right)$. We do not believe that such embeddings can be constructed "by hand". A strategy for studying symplectic embeddings of 4-dimensional ellipsoids by algebrogeometric tools is proposed in [6].

Our next goal is to represent the (normalized) Ekeland-Hofer capacities as embedding capacities. First we need some preparations.

From the above discussion of $c^{B}$ it is clear that capacities and folding also yield bounds for the functions $c^{E(1, b)}$ and $c_{E(1, b)}$. We content ourselves with noting

Lemma 3. Let $N \in \mathbb{N}$ be given. Then for $N \leq b \leq N+1$ we have

$$
c^{E(1, b)}(a)=\left\{\begin{array}{cl}
1 / b \text { for } 1 /(N+1) \leq a \leq 1 / b, \\
a \text { for } 1 / b \leq a \leq 1
\end{array}\right.
$$

and

$$
c_{E(1, b)}(a)=\left\{\begin{array}{c}
a \text { for } \quad 0<a \leq 1 / b, \\
1 / b \text { for } 1 / b \leq a \leq 1 / N,
\end{array}\right.
$$

see Figure 2.

REMARK. Note that (4-4) completely describes $c_{E(1, b)}$ on the whole interval $(0,1]$ for $1 \leq b \leq 2$.

PROOF. As both formulas are proved similarly, we only prove (4-3). The first Ekeland-Hofer capacity gives the lower bound $c^{E(1, b)}(a) \geq a$ for all $a \in(0,1]$. Note that for $a \geq 1 / b$ this bound is achieved by the standard embedding, so that the second claim follows.

For $1 /(N+1) \leq a \leq 1 / N$ we have $\bar{c}_{N+1}(E(a, 1))=1$ and $\bar{c}_{N+1}(E(1, b))=$ $b$. By Fact 1 we see that $c^{E(1, b)} \geq 1 / b$ on this interval, and this bound is again achieved by the standard embedding. This completes the proof of (4-3). 


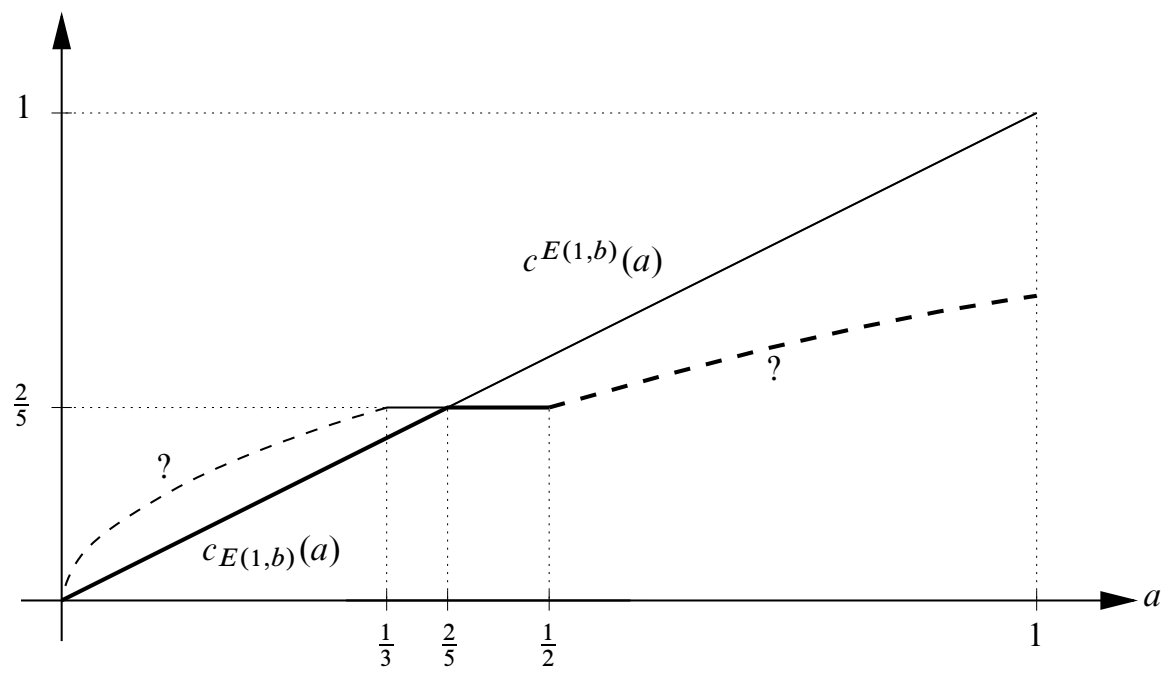

Figure 2. The functions $c^{E(1, b)}(a)$ and $c_{E(1, b)}(a)$ for $b=\frac{5}{2}$.

REMARK. Consider the functions

$$
e^{b}(a):=c^{E(1, b)}(a), \quad a \in(0,1], b \geq 1 .
$$

Notice that $e^{1}=c^{B}$. By Gromov's Nonsqueezing Theorem and monotonicity,

$$
a=c_{B}(a)=c^{Z}(a) \leq e^{b}(a) \leq c^{B}(a), \quad a \in(0,1], b \geq 1 .
$$

Since $e^{b}(a)=\left(c_{E(a, 1)}(E(1, b))\right)^{-1}$ by equation (3-1), we see that for each $a \in$ $(0,1]$ the function $b \mapsto e^{b}(a)$ is monotone decreasing and continuous. By (4-3), it satisfies $e^{b}(a)=a$ for $a \geq 1 / b$. In particular, we see that the family of graphs $\left\{\operatorname{graph}\left(e^{b}\right) \mid 1 \leq b<\infty\right\}$ fills the whole region between the graphs of $c_{B}$ and $c^{B}$; see Figure 1 .

The normalized Ekeland-Hofer capacities are represented by piecewise linear functions $\bar{c}_{k}(a)$. Indeed, $\bar{c}_{1}(a)=a$ for all $a \in(0,1]$, and for $k \geq 2$ the following formula follows straight from the definition.

Lemma 4. Setting $m:=\left[\frac{k+1}{2}\right]$, the function $\bar{c}_{k}:(0,1] \rightarrow(0,1]$ is given by

$$
\bar{c}_{k}(a)=\left\{\begin{array}{rlrl}
\frac{k+1-i}{m} a & \text { for } \frac{i-1}{k+1-i} & \leq a \leq \frac{i}{k+1-i}, \\
\frac{i}{m} & \text { for } \frac{i}{k+1-i} \leq a \leq \frac{i}{k-i} .
\end{array}\right.
$$

Here $i$ takes integer values between 1 and $m$.

Figure 3 shows the first six of the $\bar{c}_{k}$ and their limit function $c_{\infty}$ according to Proposition 1. 


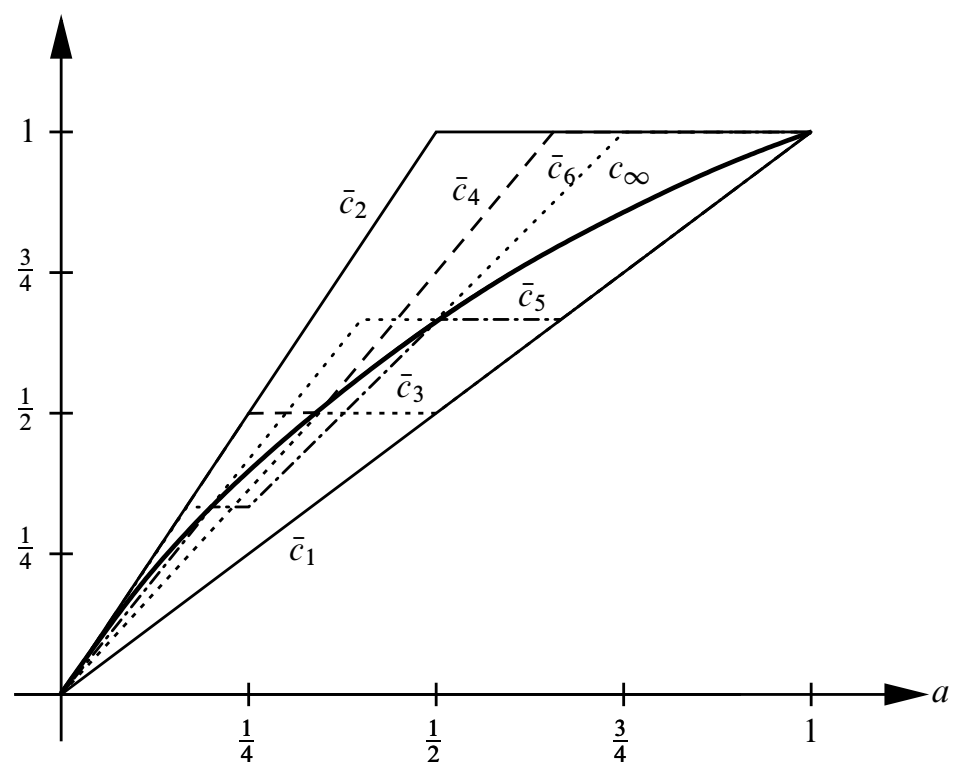

Figure 3. The first six $\bar{c}_{k}$ and $c_{\infty}$.

In dimension 4 , the uniform convergence $\bar{c}_{k} \rightarrow c_{\infty}$ is very transparent, as can be seen in Figure 3. One readily checks that $\bar{c}_{k}-c_{\infty} \geq 0$ if $k$ is even, in which case $\left\|\bar{c}_{k}-c_{\infty}\right\|=\frac{1}{k+1}$, and that $\bar{c}_{k}-c_{\infty} \leq 0$ if $k=2 m-1$ is odd, in which case $\left\|\bar{c}_{k}-c_{\infty}\right\|=\frac{m-1}{m k}$ if $k \geq 3$. Note that the sequences of the even (resp. odd) $\bar{c}_{k}$ are almost, but not quite, decreasing (resp. increasing). We still have

COROLlaRY 1. For all $r, s \in \mathbb{N}$, we have

$$
\bar{c}_{2 r s} \leq \bar{c}_{2 r} .
$$

This will be a consequence of the following characterization of Ekeland-Hofer capacities.

Lemma 5. Fix $k \in \mathbb{N}$ and denote by $\left[a_{l}, b_{l}\right]$ the interval on which $\bar{c}_{k}$ has the value $l /\left[\frac{k+1}{2}\right]$. Then

(a) $\bar{c}_{k} \leq c$ for every capacity $c$ such that $\bar{c}_{k}\left(a_{l}\right) \leq c\left(a_{l}\right)$ for all $l=1,2, \ldots,\left[\frac{k+1}{2}\right]$.

(b) $\bar{c}_{k} \geq c$ for every capacity $c$ such that $\bar{c}_{k}\left(b_{l}\right) \geq c\left(b_{l}\right)$ for all $l=1,2, \ldots,\left[\frac{k}{2}\right]$ and

$$
\lim _{a \rightarrow 0} \frac{c(a)}{a} \leq \frac{k}{\left[\frac{k+1}{2}\right]} .
$$

PROOF. Formula (4-2) and Lemma 4 show that where a normalized EkelandHofer capacity grows, it grows with maximal slope. In particular, going left from the left end point $a_{l}$ of a plateau a normalized Ekeland-Hofer capacity drops 
with the fastest possible rate until it reaches the level of the next lower plateau and then stays there, showing the minimality. Similarly, going right from the right end point $b_{l}$ of some plateau a normalized Ekeland-Hofer capacity grows with the fastest possible rate until it reaches the next higher level, showing the maximality.

PROOF OF COROLlaRY 1. The right end points of plateaus for $\bar{c}_{2 r}$ are given by $b_{i}=\frac{i}{2 r-i}$. Thus we compute

$$
\bar{c}_{2 r}\left(\frac{i}{2 r-i}\right)=\frac{i}{r}=\frac{i s}{r s}=\bar{c}_{2 r s}\left(\frac{i s}{2 r s-i s}\right)=\bar{c}_{2 r s}\left(\frac{i}{2 r-i}\right)
$$

and the claim follows from the characterization of $\bar{c}_{2 r}$ by maximality.

Lemma 3 and the piecewise linearity of the $\bar{c}_{k}$ suggest that they may be representable as embedding capacities into a disjoint union of finitely many ellipsoids. This is indeed the case.

PROPOSITION 2. The normalized Ekeland-Hofer capacity $\bar{c}_{k}$ on Ell ${ }^{4}$ is the capacity $c^{X_{k}}$ of embeddings into the disjoint union of ellipsoids

$$
X_{k}=Z\left(\frac{m}{k}\right) \amalg \coprod_{j=1}^{\left[\frac{k}{2}\right]} E\left(\frac{m}{k-j}, \frac{m}{j}\right),
$$

where $m=\left[\frac{k+1}{2}\right]$.

PROOF. The proposition clearly holds for $k=1$. We thus fix $k \geq 2$. Recall from Lemma 4 that $\bar{c}_{k}$ has $\left[\frac{k}{2}\right]$ plateaus, the $j$-th of which has height $\frac{j}{m}$ and starts at $a_{j}:=j /(k+1-j)$ and ends at $b_{j}:=j /(k-j)$. The $j$-th ellipsoid in Proposition 2 is found as follows: In view of (4-3) we first select an ellipsoid $E(1, b)$ so that the point $\frac{1}{b}$ corresponds to $b_{j}$. This ellipsoid is then rescaled to achieve the correct height $\frac{j}{m}$ of the plateau (note that by conformality, $\alpha c^{E(\alpha, \alpha b)}=c^{E(1, b)}$ for $\alpha>0$ ). We obtain the candidate ellipsoid

$$
E_{j}=E\left(\frac{m}{k-j}, \frac{m}{j}\right) .
$$

The slope of $\bar{c}_{k}$ following its $j$-th plateau and the slope of $c^{E_{j}}$ after its plateau both equal $\frac{k-j}{m}$. The cylinder is added to achieve the correct behavior near $a=0$. We are thus left with showing that for each $1 \leq j \leq\left[\frac{k}{2}\right]$,

$$
\bar{c}_{k}(a) \leq c^{E_{j}}(a) \quad \text { for all } a \in(0,1]
$$


According to Lemma 5 (a) it suffices to show that for each $1 \leq j \leq\left[\frac{k}{2}\right]$ and each $1 \leq l \leq\left[\frac{k}{2}\right]$ we have

$$
\bar{c}_{k}\left(a_{l}\right)=\frac{l}{m} \leq c^{E_{j}}\left(a_{l}\right)
$$

For $l>j$, the estimate (4-6) follows from the fact that $\bar{c}_{k}=c^{E_{j}}$ near $b_{j}$ and from the argument given in the proof of Lemma 5 (a), and for $l=j$ the estimate (4-6) follows from (4-3) of Lemma 3 by a direct computation. We will deal with the other cases

$$
1 \leq l<j \leq\left[\frac{k}{2}\right]
$$

by estimating $c^{E_{j}}\left(a_{l}\right)$ from below, using Fact 1 with $c=c_{\mathrm{vol}}$ and $c=\bar{c}_{2}$.

Fix $j$ and recall that $c_{\mathrm{vol}}(E(x, y))=\sqrt{x y}$, so

$$
c^{E_{j}}\left(a_{l}\right) \geq \frac{c_{\mathrm{vol}}\left(E\left(a_{l}, 1\right)\right)}{c_{\mathrm{vol}}\left(E\left(\frac{m}{k-j}, \frac{m}{j}\right)\right)}=\sqrt{\frac{l j(k-j)}{(k+1-l) m^{2}}}=\frac{l}{m} \cdot \sqrt{\frac{j(k-j)}{(k+1-l) l}}
$$

gives the desired estimate (4-6) if $j(k-j) \geq-l^{2}+(k+1) l$. Computing the roots $l_{ \pm}$of this quadratic inequality in $l$, we find that this is the case if

$$
l \leq l_{-}=\frac{1}{2}\left(k+1-\sqrt{1+2 k+(k-2 j)^{2}}\right) .
$$

Computing the normalized second Ekeland-Hofer capacity under the assumption that $a_{l} \leq \frac{1}{2}$, we find that $\bar{c}_{2}\left(E\left(a_{l}, 1\right)\right)=2 a_{l}=\frac{2 l}{k+1-l}$ and $\bar{c}_{2}\left(E_{j}\right) \leq \frac{m}{j}$, so

$$
c^{E_{j}}\left(a_{l}\right) \geq \frac{\bar{c}_{2}\left(E\left(a_{l}, 1\right)\right)}{\bar{c}_{2}\left(E\left(\frac{m}{k-j}, \frac{m}{j}\right)\right)} \geq \frac{2 l}{k+1-l} \cdot \frac{j}{m}=\frac{l}{m} \cdot \frac{2 j}{k+1-l},
$$

which gives the required estimate (4-6) if

$$
l \geq k+1-2 j .
$$

Note that for $\frac{1}{2} \leq a_{l} \leq 1$ we have $\bar{c}_{2}\left(E\left(a_{l}, 1\right)\right)=1$ and hence

$$
\frac{\bar{c}_{2}\left(E\left(a_{l}, 1\right)\right)}{\bar{c}_{2}\left(E\left(\frac{m}{k-j}, \frac{m}{j}\right)\right)} \geq \frac{j}{m}>\frac{l}{m}
$$

trivially, because we only consider $l<j$. 
So combining the results from the two capacities, we find that the desired estimate (4-6) holds provided either $l \leq l_{-}=\frac{1}{2}\left(k+1-\sqrt{1+2 k+(k-2 j)^{2}}\right)$ or $l \geq k+1-2 j$. As we only consider $l<j$, it suffices to verify that

$$
\min (j-1, k+1-2 j) \leq \frac{1}{2}\left(k+1-\sqrt{1+2 k+(k-2 j)^{2}}\right)
$$

for all positive integers $j$ and $k$ satisfying $1 \leq j \leq\left[\frac{k}{2}\right]$. This indeed follows from another straightforward computation, completing the proof of Proposition 2.

Using the results above, we find a presentation of the normalized capacity $c_{\infty}=$ $\lim _{k \rightarrow \infty} \bar{c}_{k}$ on $E l l^{4}$ as embedding capacity into a countable disjoint union of ellipsoids. Indeed, the space $X_{4 r}$ appearing in the statement of Proposition 2 is obtained from $X_{2} r$ by adding $r$ more ellipsoids. Combined with Proposition 1 this yields the presentation

$$
c_{\infty}=c^{X} \quad \text { on } E l l^{4},
$$

where $X=\coprod_{r=1}^{\infty} X_{2 r}$ is a disjoint union of countably many ellipsoids. Together with Conjecture 1 , the following conjecture suggests a much more efficient presentation of $c_{\infty}$ as an embedding capacity.

CONJECTURE 2. The restriction of the normalized Lagrangian capacity $\bar{c}_{L}$ to Ell ${ }^{4}$ equals the embedding capacity $c^{X}$, where $X$ is the connected subset $B(1) \cup Z\left(\frac{1}{2}\right)$ of $\mathbb{R}^{4}$.

For the embedding capacities from ellipsoids, we have the following analogue of Proposition 2.

Proposition 3. The normalized Ekeland-Hofer capacity $\bar{c}_{k}$ on Ell ${ }^{4}$ is the maximum of finitely many capacities $c_{E_{k, j}}$ of embeddings of ellipsoids $E_{k, j}$,

$$
\bar{c}_{k}(a)=\max \left\{c_{E_{k, j}}(a) \mid 1 \leq j \leq m\right\}, \quad a \in(0,1],
$$

where

with $m=\left[\frac{k+1}{2}\right]$.

$$
E_{k, j}=E\left(\frac{m}{k+1-j}, \frac{m}{j}\right)
$$

PROOF. The ellipsoids $E_{k, j}$ are determined using (4-4) in Lemma 3. According to Lemma 5 (b), this time it suffices to check that for all $1 \leq j \leq l \leq\left[\frac{k}{2}\right]$ the values of the corresponding capacities at the right end points $b_{l}=\frac{l}{k-l}$ of plateaus of $\bar{c}_{k}$ satisfy

$$
c_{E_{k, j}}\left(b_{l}\right) \leq \frac{l}{m}=\bar{c}_{k}\left(b_{l}\right)
$$


The case $l=j$ follows from (4-4) in Lemma 3 by a direct computation. For the remaining cases

$$
1 \leq j<l \leq\left[\frac{k}{2}\right]
$$

we use three different methods, depending on the value of $j$. If $j \leq \frac{k-1}{3}$, then Fact 1 with $c=c_{\text {vol }}$ gives (4-7) by a computation similar to the one in the proof of Proposition 2. If $j \geq \frac{k+1}{3}$, then $a_{j}=\frac{j}{k+1-j} \geq \frac{1}{2}$, so that (4-4) in Lemma 3 shows that $c_{E_{k, j}}$ is constant on $\left[a_{j}, 1\right]$, proving (4-7) in this case. Finally, if $j=\frac{k}{3}$ and $l \geq j+1$, then $\bar{c}_{2}\left(E_{k, j}\right)=\frac{2 m}{k+1-j}$ and $\bar{c}_{2}\left(b_{l}\right)=1$, so with Fact 1 we get

$$
c_{E_{k, j}}\left(b_{l}\right) \leq \frac{k+1-j}{2 m},
$$

which is smaller than $\frac{l}{m}$ for the values of $j$ and $l$ we consider here. This completes the proof of Proposition 3.

Here is the corresponding conjecture for the normalized Lagrangian capacity.

CONJECTURE 3. The restriction of the normalized Lagrangian capacity $\bar{c}_{L}$ to Ell $^{2 n}$ equals the embedding capacity $c_{P(1 / n, \ldots, 1 / n)}$ of the cube of radius $1 / \sqrt{n}$.

\subsection{Polydiscs.}

4.2.1. Arbitrary dimension. Again we first describe the values of the capacities in Section 2 on polydiscs.

The values of the Gromov radius $c_{B}$ on polydiscs are

$$
c_{B}\left(P\left(a_{1}, \ldots, a_{n}\right)\right)=\min \left\{a_{1}, \ldots, a_{n}\right\} .
$$

As for ellipsoids, this also determines the values of $c_{1}^{\mathrm{EH}}, c_{\mathrm{HZ}}, e\left(\cdot, \mathbb{R}^{2 n}\right)$ and $c^{Z}$. According to [19], the values of Ekeland-Hofer capacities on polydiscs are

$$
c_{k}^{\mathrm{EH}}\left(P\left(a_{1}, \ldots, a_{n}\right)\right)=k \pi \min \left\{a_{1}, \ldots, a_{n}\right\} .
$$

Using Chekanov's result [11] that $A_{\min }(L) \leq e\left(L, \mathbb{R}^{2 n}\right)$ for every closed Lagrangian submanifold $L \subset \mathbb{R}^{2 n}$, one finds the values of the Lagrangian capacity on polydiscs to be

$$
c_{L}\left(P\left(a_{1}, \ldots, a_{n}\right)\right)=\pi \min \left\{a_{1}, \ldots, a_{n}\right\} .
$$

Since $\operatorname{vol}\left(P\left(a_{1}, \ldots, a_{n}\right)\right)=a_{1} \cdots a_{n} \cdot \pi^{n}$ and $\operatorname{vol}\left(B^{2 n}\right)=\frac{\pi^{n}}{n !}$, the values of the volume capacity on polydiscs are

$$
c_{\mathrm{vol}}\left(P\left(a_{1}, \ldots, a_{n}\right)\right)=\left(a_{1} \cdots a_{n} \cdot n !\right)^{1 / n} .
$$


As in the case of ellipsoids, a (generalized) capacity $c$ on $\mathrm{Pol}^{2 n}$ can be viewed as a function

$$
c\left(a_{1}, \ldots, a_{n-1}\right):=c\left(P\left(a_{1}, \ldots, a_{n-1}, 1\right)\right)
$$

on the set $\left\{0<a_{1} \leq \ldots \leq a_{n-1} \leq 1\right\}$. Directly from the definitions and the computations above we obtain the following easy analogue of Proposition 1.

Proposition 4. As $k \rightarrow \infty$, the normalized Ekeland-Hofer capacities $\bar{c}_{k}$ converge on Pol ${ }^{2 n}$ uniformly to the normalized Lagrangian capacity $\bar{c}_{L}=n c_{L} / \pi$.

Propositions 4 and 1 (together with Conjecture 1) give rise to

PROBLEM 17. What is the largest subcategory of $O p^{2 n}$ on which the normalized Lagrangian capacity is the limit of the normalized Ekeland-Hofer capacities?

4.2.2. Polydiscs in dimension 4. Again, a normalized (generalized) capacity on polydiscs in dimension 4 is represented by a function $c(a):=c(P(a, 1))$ of a single real variable $0<a \leq 1$, which has the properties (i), (ii), (iii). Contrary to ellipsoids, these properties are not the only restrictions on a normalized capacity on 4-dimensional polydiscs even if one restricts to linear symplectic embeddings as morphisms. Indeed, the linear symplectomorphism

$$
\left(z_{1}, z_{2}\right) \mapsto \frac{1}{\sqrt{2}}\left(z_{1}+z_{2}, z_{1}-z_{2}\right)
$$

of $\mathbb{R}^{4}$ yields a symplectic embedding

$$
P(a, b) \hookrightarrow P\left(\frac{a+b}{2}+\sqrt{a b}, \frac{a+b}{2}+\sqrt{a b}\right)
$$

for any $a, b>0$, which implies

FACT 12. For any normalized capacity $c$ on LinPol ${ }^{4}$,

$$
c(a) \leq \frac{1}{2}+\frac{a}{2}+\sqrt{a} .
$$

Still, we have the following easy analogues of Propositions 2 and 3.

Proposition 5. The normalized Ekeland-Hofer capacity $\bar{c}_{k}$ on Pol ${ }^{4}$ is the capacity $c^{Y_{k}}$, where

$$
Y_{k}=Z\left(\frac{\left[\frac{k+1}{2}\right]}{k}\right),
$$

as well as the capacity $c_{Y_{k}^{\prime}}$, where

$$
Y_{k}^{\prime}=B\left(\frac{\left[\frac{k+1}{2}\right]}{k}\right) .
$$


COROLlary 2. The identity $\bar{c}_{k}=c^{X_{k}}$ of Proposition 2 extends to $\mathrm{Ell}^{4} \cup \mathrm{Pol}^{4}$.

Proof. Note that $Y_{k}$ is the first component of the space $X_{k}$ of Proposition 2. It thus remains to show that for each of the ellipsoid components $E_{j}$ of $X_{k}$,

$$
\bar{c}_{k}(P(a, 1)) \leq c^{E_{j}}(P(a, 1)), \quad a \in(0,1] .
$$

This follows at once from the observation that for each $j$ we have $c_{k}^{\mathrm{EH}}\left(E_{j}\right)=$ $\left[\frac{k+1}{2}\right] \pi$, whereas $c_{k}^{\mathrm{EH}}(P(a, 1))=k a \pi$.

PROBLEM 18. Does the equality $\bar{c}_{k}=c^{X_{k}}$ hold on a larger class of open subsets of $\mathbb{R}^{4}$ ?

\section{References}

[1] S. Bates, Some simple continuity properties of symplectic capacities, The Floer memorial volume, 185-193, Progr. Math. 133, Birkhäuser, Basel, 1995.

[2] S. Bates, A capacity representation theorem for some non-convex domains, Math. Z. 227, 571-581 (1998).

[3] P. Biran, Symplectic packing in dimension 4, Geom. Funct. Anal. 7, 420-437 (1997).

[4] P. Biran, A stability property of symplectic packing, Invent. Math. 136, 123-155 (1999).

[5] P. Biran, Constructing new ample divisors out of old ones, Duke Math. J. 98, 113135 (1999).

[6] P. Biran, From symplectic packing to algebraic geometry and back, European Congress of Mathematics, Vol. II (Barcelona, 2000), 507-524, Progr. Math. 202, Birkhäuser, Basel, 2001.

[7] P. Biran, Geometry of symplectic intersections, Proceedings of the International Congress of Mathematicians, Vol. II (Beijing, 2002), 241-255, Higher Ed. Press, Beijing, 2002.

[8] P. Biran and K. Cieliebak, Symplectic topology on subcritical manifolds, Comment. Math. Helv. 76, 712-753 (2001).

[9] P. Biran, L. Polterovich and D. Salamon, Propagation in Hamiltonian dynamics and relative symplectic homology, Duke Math. J. 119, 65-118 (2003).

[10] F. Bourgeois, Ya. Eliashberg, H. Hofer, K. Wysocki and E. Zehnder, Compactness results in symplectic field theory, Geom. Topol. 7, 799-888 (2003).

[11] Y. Chekanov, Hofer's symplectic energy and Lagrangian intersections, Contact and Symplectic Geometry, ed. C. B. Thomas, Publ. Newton Inst. 8, 296-306, Cambridge University Press, Cambridge, 1996.

[12] Y. Chekanov, Lagrangian intersections, symplectic energy, and areas of holomorphic curves, Duke Math. J. 95, 213-226 (1998).

[13] Y. Chekanov, talk on a hike on Üetliberg on a sunny day in May 2004.

[14] K. Cieliebak, A. Floer and H. Hofer, Symplectic homology. II. A general construction, Math. Z. 218, 103-122 (1995). 
[15] K. Cieliebak and E. Goldstein, A note on mean curvature, Maslov class and symplectic area of Lagrangian immersions, J. Symplectic Geom. 2, 261-266 (2004).

[16] K. Cieliebak and K. Mohnke, Punctured holomorphic curves and Lagrangian embeddings, preprint, 2003.

[17] S. Donaldson, Symplectic submanifolds and almost-complex geometry, J. Differential Geom. 44, 666-705 (1996).

[18] I. Ekeland and H. Hofer, Symplectic topology and Hamiltonian dynamics, Math. Z. 200, 355-378 (1989).

[19] I. Ekeland and H. Hofer, Symplectic topology and Hamiltonian dynamics II, Math. Z. 203, 553-567 (1990).

[20] I. Ekeland and S. Mathlouthi, Calcul numérique de la capacité symplectique, Progress in variational methods in Hamiltonian systems and elliptic equations (L'Aquila, 1990), 68-91, Pitman Res. Notes Math. Ser. 243, Longman Sci. Tech., Harlow, 1992.

[21] Y. Eliashberg, Symplectic topology in the nineties, Symplectic geometry. Differential Geom. Appl. 9, 59-88 (1998).

[22] Y. Eliashberg, A. Givental and H. Hofer, Introduction to symplectic field theory, GAFA 2000 (Tel Aviv, 1999), Geom. Funct. Anal. 2000, Special Volume, Part II, 560-673.

[23] Y. Eliashberg and M. Gromov, Convex symplectic manifolds, Several complex variables and complex geometry, Part 2 (Santa Cruz, CA, 1989), 135-162, Proc. Sympos. Pure Math. 52, Part 2, American Mathematical Society, Providence, RI, 1991.

[24] Y. Eliashberg and H. Hofer, Unseen symplectic boundaries, Manifolds and geometry, Sympos. Math. XXXVI (Pisa, 1993) 178-189, Cambridge Univ. Press, Cambridge, 1996.

[25] Y. Eliashberg and H. Hofer, An energy-capacity inequality for the symplectic holonomy of hypersurfaces flat at infinity, Symplectic geometry, 95-114, London Math. Soc. Lecture Note Ser. 192, Cambridge Univ. Press, Cambridge, 1993.

[26] E. Fadell and P. Rabinowitz, Generalized cohomological index theories for Lie group actions with an application to bifurcation questions for Hamiltonian systems, Invent. Math. 45, 139-173 (1978).

[27] A. Floer, H. Hofer and C. Viterbo, The Weinstein conjecture in $P \times \mathbb{C}^{l}$, Math. Z. 203, 469-482 (1990).

[28] A Floer, H. Hofer, Symplectic homology. I. Open sets in $\mathbb{C}^{n}$, Math. Z. 215, 37-88 (1994).

[29] A Floer, H. Hofer and K. Wysocki, Applications of symplectic homology. I, Math. Z. 217, 577-606 (1994).

[30] U. Frauenfelder, V. Ginzburg and F. Schlenk, Energy capacity inequalities via an action selector, Geometry, spectral theory, groups, and dynamics, 129-152, Contemp. Math. 387, Amer. Math. Soc., Providence, RI, 2005.

[31] U. Frauenfelder and F. Schlenk, Hamiltonian dynamics on convex symplectic manifolds, Israel J. of Maths. 159, 1-56 (2007). 
[32] V. Ginzburg, An embedding $S^{2 n-1} \rightarrow \mathbf{R}^{2 n}, 2 n-1 \geq 7$, whose Hamiltonian flow has no periodic trajectories, Internat. Math. Res. Notices 1995, 83-97 (1995).

[33] V. Ginzburg, A smooth counterexample to the Hamiltonian Seifert conjecture in $\mathbf{R}^{6}$, Internat. Math. Res. Notices 1997, 641-650 (1997).

[34] V. Ginzburg, The Weinstein conjecture and theorems of nearby and almost existence, The breadth of symplectic and Poisson geometry, 139-172, Progr. Math. 232, Birkhäuser, Boston, MA, 2005.

[35] V. Ginzburg and B. Gürel, $A C^{2}$-smooth counterexample to the Hamiltonian Seifert conjecture in $\mathbb{R}^{4}$, Ann. of Math. (2) 158, 953-976 (2003).

[36] V. Ginzburg and B. Gürel, Relative Hofer-Zehnder capacity and periodic orbits in twisted cotangent bundles, Duke Math. J. 123, 1-47 (2004).

[37] V. Ginzburg and E. Kerman, Periodic orbits in magnetic fields in dimensions greater than two, Geometry and topology in dynamics (Winston-Salem, NC, 1998, and San Antonio, TX, 1999), 113-121, Contemp. Math. 246, American Mathematical Society, Providence, RI, 1999.

[38] E. Goldstein, Some estimates related to Oh's conjecture for the Clifford tori in $\mathbb{C P}^{n}$, preprint, math.DG/0311460.

[39] M. Gromov, Pseudo holomorphic curves in symplectic manifolds, Invent. Math. 82, 307-347 (1985).

[40] D. Hermann, Holomorphic curves and Hamiltonian systems in an open set with restricted contact-type boundary, Duke Math. J. 103, 335-374 (2000).

[41] D. Hermann, Inner and outer Hamiltonian capacities, Bull. Soc. Math. France 132, 509-541 (2004).

[42] D. Hermann, Symplectic capacities and symplectic convexity, Preprint, 2005.

[43] H. Hofer, On the topological properties of symplectic maps, Proc. Roy. Soc. Edinburgh Sect. A 115, 25-38 (1990).

[44] H. Hofer, Symplectic capacities, Geometry of low-dimensional manifolds, 2 (Durham, 1989), 15-34, London Math. Soc. Lecture Note Ser. 151, Cambridge Univ. Press, Cambridge, 1990.

[45] H. Hofer, Estimates for the energy of a symplectic map, Comment. Math. Helv. 68, 48-72 (1993).

[46] H. Hofer, Pseudoholomorphic curves in symplectizations with applications to the Weinstein conjecture in dimension three, Invent. Math. 114, 515-563 (1993).

[47] H. Hofer and C. Viterbo, The Weinstein conjecture in the presence of holomorphic spheres, Comm. Pure Appl. Math. 45, 583-622 (1992).

[48] H. Hofer and E. Zehnder, A new capacity for symplectic manifolds, Analysis, et cetera, 405-427, Academic Press, Boston, MA, 1990.

[49] H. Hofer and E. Zehnder, Symplectic Invariants and Hamiltonian Dynamics, Birkhäuser, Basel (1994).

[50] H. Iriyeh, H. Ono and T. Sakai, Integral geometry and Hamiltonian volume minimizing property of a totally geodesic Lagrangian torus in $S^{2} \times S^{2}$, Proc. Japan Acad. Ser. A Math. Sci. 79, 167-170 (2003).

[51] M.-Y. Jiang, Hofer-Zehnder symplectic capacity for two-dimensional manifolds, Proc. Roy. Soc. Edinburgh Sect. A 123, 945-950 (1993). 
[52] M.-Y. Jiang, Symplectic embeddings from $\mathbf{R}^{2 n}$ into some manifolds, Proc. Roy. Soc. Edinburgh Sect. A 130, 53-61 (2000).

[53] B. Kruglikov, A remark on symplectic packings, Dokl. Akad. Nauk 350, 730-734 (1996).

[54] F. Lalonde, Energy and capacities in symplectic topology, Geometric topology (Athens, GA, 1993), 328-374, AMS/IP Stud. Adv. Math. 2.1, American Mathematical Society, Providence, RI, 1997.

[55] F. Lalonde and D. McDuff, The geometry of symplectic energy, Ann. of Math. (2) 141, 349-371 (1995).

[56] F. Lalonde and D. McDuff, Hofer's $L^{\infty}$-geometry: energy and stability of Hamiltonian flows. I, II, Invent. Math. 122, 1-33, 35-69 (1995).

[57] F. Lalonde and C. Pestieau, Stabilisation of symplectic inequalities and applications, Northern California Symplectic Geometry Seminar, 63-71, AMS Transl. Ser. 2 196, American Mathematical Society, Providence, RI, 1999.

[58] F. Lalonde and M. Pinsonnault, The topology of the space of symplectic balls in rational 4-manifolds, Duke Math. J. 122, 347-397 (2004).

[59] G. Lu, The Weinstein conjecture on some symplectic manifolds containing the holomorphic spheres, Kyushu J. Math. 52, 331-351 (1998) and 54, 181-182 (2000).

[60] G. Lu, Symplectic capacities of toric manifolds and combinatorial inequalities, C. R. Math. Acad. Sci. Paris 334, 889-892 (2002).

[61] L. Macarini, Hofer-Zehnder capacity and Hamiltonian circle actions, Commun. Contemp. Math. 6, 913-945 (2004).

[62] L. Macarini and F. Schlenk, A refinement of the Hofer-Zehnder theorem on the existence of closed trajectories near a hypersurface, Bull. London Math. Soc. 37, 297-300 (2005).

[63] F. Maley, J. Mastrangeli, L. Traynor, Symplectic packings in cotangent bundles of tori, Experiment. Math. 9, 435-455 (2000).

[64] D. McDuff, Blowing up and symplectic embeddings in dimension 4, Topology $\mathbf{3 0}$, 409-421 (1991).

[65] D. McDuff, Symplectic manifolds with contact type boundaries, Invent. Math. 103, 651-671 (1991).

[66] D. McDuff, Symplectic topology and capacities, Prospects in mathematics (Princeton, NJ, 1996), 69-81, American Mathematical Society, Providence, RI, 1999.

[67] D. McDuff, Geometric variants of the Hofer norm, J. Symplectic Geom. 1, 197252 (2002).

[68] D. McDuff and L. Polterovich, Symplectic packings and algebraic geometry, Invent. Math. 115, 405-429 (1994).

[69] D. McDuff and D. Salamon, Introduction to symplectic topology, Second edition, Oxford Mathematical Monographs, The Clarendon Press, Oxford University Press, New York, 1998.

[70] D. McDuff and D. Salamon, $J$-holomorphic curves and symplectic topology, AMS Colloquium Publications 52, American Mathematical Society, Providence, RI, 2004.

[71] D. McDuff and J. Slimowitz, Hofer-Zehnder capacity and length minimizing Hamiltonian paths, Geom. Topol. 5, 799-830 (2001). 
[72] D. McDuff and L. Traynor, The 4-dimensional symplectic camel and related results, Symplectic geometry, 169-182, London Math. Soc. Lecture Note Ser. 192, Cambridge Univ. Press, Cambridge (1993).

[73] E. Neduv, Prescribed minimal period problems for convex Hamiltonian systems via Hofer-Zehnder symplectic capacity, Math. Z. 236, 99-112 (2001).

[74] Y.-G. Oh, Second variation and stabilities of minimal Lagrangian submanifolds in Kähler manifolds, Invent. Math. 101, 501-519 (1990).

[75] Y.-G. Oh, Chain level Floer theory and Hofer's geometry of the Hamiltonian diffeomorphism group, Asian J. Math. 6, 579-624 (2002).

[76] Y.-G. Oh, Construction of spectral invariants of Hamiltonian paths on closed symplectic manifolds, The breadth of symplectic and Poisson geometry, 525-570, Progr. Math. 232, Birkhäuser Boston, Boston, MA, 2005.

[77] Y.-G. Oh, Spectral invariants, analysis of the Floer moduli space, and geometry of the Hamiltonian diffeomorphism group, Duke Math. J. 130, 199-295 (2005).

[78] E. Opshtein, Maximal symplectic packings in $\mathscr{P}^{2}$, To appear in Compos. Math.

[79] L. Polterovich, Gromov's K-area and symplectic rigidity, Geom. Funct. Anal. 6, 726-739 (1996).

[80] L. Polterovich, Symplectic aspects of the first eigenvalue, J. Reine Angew. Math. 502, 1-17 (1998).

[81] L. Polterovich, Hamiltonian loops from the ergodic point of view, J. Eur. Math. Soc. 1, 87-107 (1999).

[82] L. Polterovich, The geometry of the group of symplectic diffeomorphisms, Lectures in Mathematics ETH Zürich, Birkhäuser, Basel, 2001.

[83] P. Rabinowitz, Periodic solutions of Hamiltonian systems, Comm. Pure Appl. Math. 31, 157-184 (1978).

[84] P. Rabinowitz, Periodic solutions of a Hamiltonian system on a prescribed energy surface, J. Differential Equations 33, 336-352 (1979).

[85] F. Schlenk, Symplectic embedding of ellipsoids, Israel J. of Math. 138, 215-252 (2003).

[86] F. Schlenk, Embedding problems in symplectic geometry, De Gruyter Expositions in Mathematics 40, Walter de Gruyter, Berlin, 2005.

[87] F. Schlenk, Applications of Hofer's geometry to Hamiltonian dynamics, Comment. Math. Helv. 81, 105-121 (2006).

[88] M. Schwarz, On the action spectrum for closed symplectically aspherical manifolds, Pacific J. Math. 193, 419-461 (2000).

[89] K.-F. Siburg, Symplectic capacities in two dimensions, Manuscripta Math. 78, 149-163 (1993).

[90] J.-C. Sikorav, Systèmes Hamiltoniens et topologie symplectique, Dipartimento di Matematica dell' Università di Pisa, ETS Editrice, Pisa, 1990.

[91] J.-C. Sikorav, Quelques propriétés des plongements lagrangiens, Analyse globale et physique mathématique (Lyon, 1989), Mém. Soc. Math. France 46, 151-167 (1991).

[92] T. Tokieda, Isotropic isotopy and symplectic null sets, Proc. Nat. Acad. Sci. U.S.A. 94, 13407-13408 (1997). 
[93] L. Traynor, Symplectic homology via generating functions, Geom. Funct. Anal. 4, 718-748 (1994).

[94] L. Traynor, Symplectic packing constructions, J. Differential Geom. 42, 411-429 (1995).

[95] C. Viterbo, Capacités symplectiques et applications (d'après Ekeland-Hofer, Gromov), Séminaire Bourbaki, Vol. 1988/89. Astérisque 177-178 (1989), Exp. No. 714, 345-362.

[96] C. Viterbo, Plongements lagrangiens et capacités symplectiques de tores dans $\mathbb{R}^{2 n}$, C. R. Acad. Sci. Paris Sér. I Math. 311, 487-490 (1990).

[97] C. Viterbo, Symplectic topology as the geometry of generating functions, Math. Ann. 292, 685-710 (1992).

[98] C. Viterbo, Metric and isoperimetric problems in symplectic geometry, J. Amer. Math. Soc. 13, 411-431 (2000).

\author{
Kai Cieliebak \\ MATHEMATISCHES INSTITUT \\ LUDWIG-MAXIMILIANS-UNIVERSITÄT MÜNCHEN \\ THERESIENSTRASSE 39 \\ D-80333 MÜNCHEN \\ GERMANY \\ kai@mathematik.uni-muenchen.de \\ HELMUT HOFER \\ COURANT INSTITUTE \\ NEW YORK UNIVERSITY \\ 251 MERCER STREET \\ NEW YORK, NY 10012 \\ UNITED STATES \\ hofer@cims.nyu.edu \\ JANKO LATSCHEV \\ MATHEMATISCHES INSTITUT \\ LUdWIG-MAXIMILIANS-UNIVERSITÄT MÜNCHEN \\ THERESIENSTRASSE 39 \\ D-80333 MÜNCHEN \\ GERMANY \\ latschev@mathematik.uni-muenchen.de \\ FELIX SCHLENK \\ DÉPARTEMENT DE MathématiQues \\ UNIVERSITÉ LIBRE DE BRUXELLES \\ BOULEVARD DU TRIOMPHE \\ 1050 BRUXELLES \\ BELGIUM \\ fschlenk@ulb.ac.be
}

